\title{
Integrative time-scale and multi-omic analysis of host- responses to Hop stunt viroid infection
}

\author{
Joan Márquez-Molins ${ }^{1,2 *}$, Pascual Villalba-Bermell ${ }^{1 *}$, Julia Corell-Sierra ${ }^{1}$, Vicente \\ Pallás ${ }^{2}$ and Gustavo Gómez ${ }^{1}$.
}

1 Institute for Integrative Systems Biology (I2SysBio), Consejo Superior de Investigaciones Científicas (CSIC) - Universitat de València (UV), Parc Científic, Cat. Agustín Escardino 9, 46980 Paterna, Spain.

2 Instituto de Biología Molecular y Celular de Plantas (IBMCP), Consejo Superior de Investigaciones Científicas (CSIC) - Universitat Politècnica de València, CPI 8E, Av. de los Naranjos s/n, 46022 Valencia, Spain.

(* equal contribution)

\section{Correspondence}

Gustavo G. Gomez, Instituto de Biología Integrativa de Sistemas (I²SysBio), Consejo Superior de Investigaciones Científicas (CSIC) - Universitat de València (UV), Parc Científic UV, Catedrático Agustín Escardino 9, Paterna, 46980 Valencia, Spain.

Email: gustavo.gomez@csic.es

Running title

Host response to viroid infection: A new viewing

\section{Keywords:}

Viroid-host interactions, epigenetic epidemiology, host transcriptional regulation in response to pathogens, global response to biotic stress, systems biology and diseases 

made available under aCC-BY-ND 4.0 International license.

\begin{abstract}
Constricted by an extreme biological simplicity, viroids are compelled to subvert host regulatory networks in order to accomplish their infectious process. Most of the studies focused on the response to viroid infection have only addressed a specific host regulatory level and considered a unique infection time. Thus, much remains to be done if we want to understand the temporal evolution and complex nature of viroid-host interactions. Here we present an integrative analysis of the timing and intensity of the genome-wide alterations in cucumber plants infected with Hop stunt viroid (HSVd). Differential host transcriptome, sRNAnome and methylome were integrated to determine the temporal response to viroid-infection. Our results support that HSVd promotes a dynamic redesign of the cucumber regulatory pathways predominantly affecting specific regulatory layers at different infection phases. Remarkably, the initial response was characterized by a reconfiguration of the host transcriptome by differential exon usage, followed by a predominant down-regulation of the transcriptional activity possibly modulated by the host epigenetic changes associated to infection and characterized by an increased hypermethylation. The silencing of at least three cucumber transcripts potential targets of vdsRNAs was also observed. The alteration in host sRNA and miRNA metabolism was marginal. We expect that these data constituting the first comprehensive map of the cucumber-response to HSVd could contribute to elucidate the molecular basis of the host alterations triggered by viroid infection.
\end{abstract}




\section{INTRODUCTION}

Viroids (the smallest infectious agents known to date) comprise an intriguing group of plant pathogens affecting a varied range of hosts worldwide. These sub-viral pathogens are classified into two families, Pospiviroidae whose replication takes place in the nucleus, and the Avsunviroidae, that replicates in the chloroplast (1). As naked circular viroids are compelled to closely interact with diverse (many yet unknown) host factors in order to fulfil their infectious cycle in the infected plants (2-4). However, how these pathogenic RNAs alter the host development and physiology inducing disease symptoms is still an unsolved question $(3,5,6)$.

In general, plants respond to stress conditions (including pathogen infection) through a complex reprogramming of their transcriptional activity. Gene regulation is a complex process modulated by a series of coordinated events involving multiple control layers such as: epigenetic modifications, modulation of the transcriptional activity and small RNA interference (7-9). These different regulatory levels have been categorized as stress-responsive structures termed as Environmental Gene Regulatory Influence Networks (EGRINs) (10).

Increasing evidence indicate that viroids can subvert different host-EGRINs and consequently promote the emergence of the phenotypic alterations recognized (in certain viroid-host interactions) as symptoms. In this sense, diverse studies have provided support to the notion that during the infectious process, viroids promote significant alterations in diverse plant regulatory pathways $(6,11)$.

Although it has been proposed that viroids (presumably because of the highly compacted and structured genomic circular RNA), are resistant to degradation mediated by RNA-silencing (12-14), early reports evidenced that viroid-derived sRNAs (vd-sRNAs) (probably arising from the DCL-mediated processing of replication intermediates), are detected in plants infected by members of both nuclear and chloroplastic families (15-17). The possibility that the vd-sRNAs could guide the silencing of plant-endogenous transcripts triggering the induction of disease symptoms, was then proposed as a plausible hypothesis to explain the basis of viroid-induced pathogenesis (17-19). Several research groups have demonstrated in the last years the involvement of vd-sRNAs in the down-regulation of host transcripts (20-28), thus emphasizing the role of RNA silencing in viroid-induced symptoms. In addition, it has been recently proposed that viroid infection might trigger the production of secondary siRNAs able to recognize host transcripts that are not direct targets of the vd-sRNAs (21). Also related to viroid-pathogenesis and RNA-silencing, alterations in the accumulation of certain endogenous miRNAs have been reported in diverse viroid-host interactions $(27,29-35)$. 
Modulation (in both positive and/or negative sense) of the transcriptional activity constitutes the core of the plant response to abiotic and biotic stress conditions $(36,37)$. Changes at the transcriptomic level have been described in several plants infected by viroids of both Pospiviroidae and Avsunviroidae families (38-44). Altogether, these studies revealed a global alteration in the expression of host genes related to defense, response to stress, hormone homeostasis and signaling, biosynthesis of cell wall compounds, and RNA metabolism, among others (11).

A growing body of evidence points toward the existence of a close interplay between infection and epigenetic alterations in the host (plant or animal) genome $(9,45)$. Consequently, epigenetic epidemiology has emerged as a promising area for future research on infectious diseases (46). Recent studies have evidenced dynamic changes in host DNA methylation patterns (mainly rDNA and transposable elements) upon viroid infection in both somatic (47, 48) and reproductive (49) tissues. The changes of the host-genome epigenetic regulation have been connected (in infected cucumber plants) with viroid recruiting and functionally subverting the host HISTONE DEACETYLASE 6 (HDA6) (48). Alteration of the host plant methylome has been also described to occur in response to infection by viruses (50) and bacteria (51), supporting that regulation of host DNA methylation may be part of an evolutionary conserved immune response in plants (52).

The exposed above evidenced that the advent of the high throughput technologies has favored the study of the viroid-host interactions at a molecular level. However, such experimental approaches have been generally centered on the analysis of the viroid-induced changes at a single host-regulatory level and/or considering a particular infection time (mainly correlated to advanced disease stages). Therefore, our knowledge about the functional diversity and temporal evolution of the global plant response to viroid infection is scarce (3).

Here we have performed an integrative analysis of the timing and intensity of the genome-wide alterations in cucumber (Cucumis sativus) plants in response to Hop stunt viroid (HSVd) infection (53). Our study has been focused on the temporal evolution (at three infection time-points) of the plant response to viroid infection at three different regulatory levels of the gene expression network: i) small RNA interference, ii) modulation of the transcriptional activity and iii) epigenetic modifications. The obtained results evidenced that HSVd infection promotes a dynamic redesign of the cucumber regulatory pathways that affects these regulatory layers distinctively depending on the infection phase. 
bioRxiv preprint doi: https://doi.org/10.1101/2022.01.06.475203; this version posted January 6, 2022. The copyright holder for this preprint (which was not certified by peer review) is the author/funder, who has granted bioRxiv a license to display the preprint in perpetuity. It is made available under aCC-BY-ND 4.0 International license.

\section{RESULTS}

\section{Viroid infection}

To uncover the dynamic of the host regulatory response to HSVd in cucumber we analyzed apical leaves of infected plants at 10 (T1), 17 (T2) and 24 (T3) days post inoculation (dpi). Mockinoculated plants were used as control. Time points were arbitrarily selected as representative of the early, intermediate and late HSVd infection phases, respectively. As expected, viroid induced symptoms were evident in HSVd-infected plants at 24 dpi (Figure 1A). A total of 54 libraries (18 for each SRNAs, long transcripts and methyl-C analysis) consisting of three bio replicates per time point of infected and control plants were obtained (Supplemental Figure S1). The information about number and quality of the obtained reads is detailed in the Supplemental Table S2 for each omic approach respectively. Sequences used in this study are available at NCBI repository (Provisional Submission code SUB10896353).

A

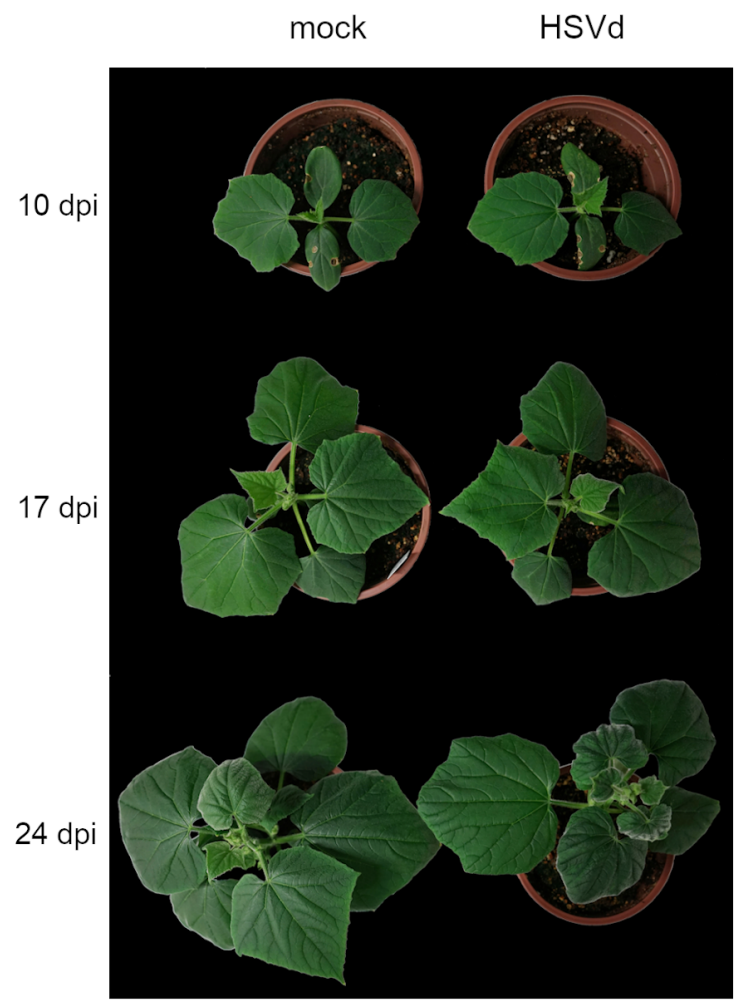

B

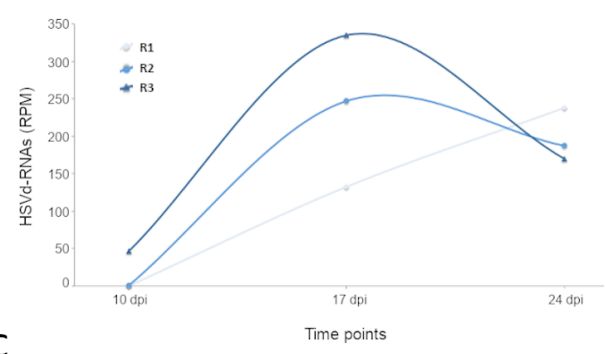

C

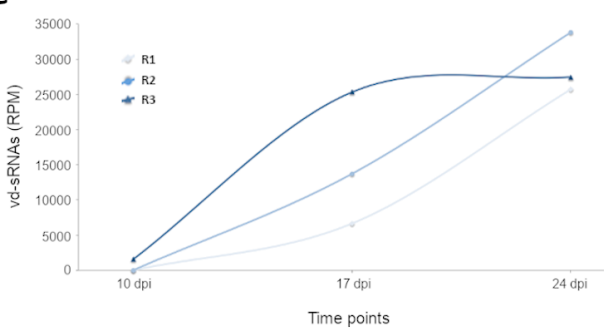

D

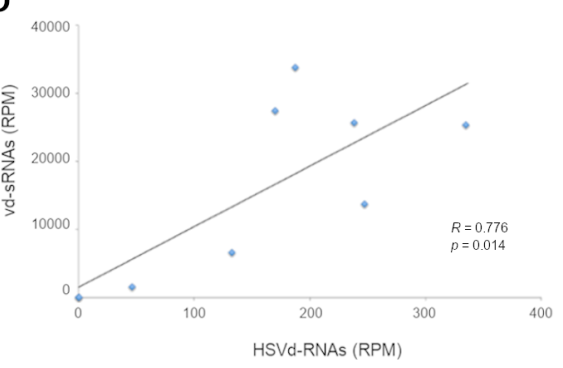

Figure 1: HSVd RNA is detected at $10 \mathrm{dpi}$ in infected plants: A) Representative infected and mock inoculated cucumber plants at the three analyzed time points. Typical plant symptoms characterized by reduction in leaf size and incipient stunting are evident at $24 \mathrm{dpi}$. Graphic representation of the total transcripts (B) and sRNAs (C) derived from HSVd genome detected in apical leaves at 10, 17 and 24 dpi. D) Scatter plot showing the significant positive correlation (estimated by Pearson correlation coefficient) between the temporal accumulation of vd-transcript and viroid sRNAs (vd-sRNAs) in infected cucumber plants.

The infection rate of inoculated plants was estimated by considering the accumulation of viroid transcripts and viroid-derived sRNAs (vd-sRNAs) (Figure 1B and C). Viroid transcripts were 
detected in two (HSVd-R2 - 0.53 RPM and HSVd-R3 - 46.4 RPM) of the three analyzed samples at $10 \mathrm{dpi}$ (T1), while at T2 (17 dpi) an increased number of HSVd-related transcripts were recovered from all the analyzed samples (132.48, 247.13 and 335.04 RPM for replicates HSVdR1, HSVd-R2 and HSVd-R3 respectively) (Supplemental Table S3). A lower accumulation (in comparison to T2) of viroid-transcripts was observed in HSVd-R2 (187.3 RPM) and HSVd-R3 (169.4 RPM) samples at T3. In contrast, the biological replicate HSVd-R1 (with no-detected viroid transcripts at T1), showed an increased accumulation (238.0 RPM) at T3 (Supplemental Table S3). On the other hand, vd-sRNAs were only detected (1421.0 RPMs) in systemic tissues of the replicate HSVd-R3 at T1 (10 dpi). The accumulation of sRNAs homologous to HSVd in infected tissues at intermediate and late HSVd infection phases was more homogenous and vdsRNAs were recovered from the three analyzed replicates at 17 dpi (6456.6 RPM, 13504.1 RPM and 25128.6 RPM) and 24 dpi (25539.9 RPM, 33627.7 RPM and 27284.7 RPM), revealing that vd-sRNAs increased through the analyzed period (Figure 1C). In general, vd-sRNAs accumulation correlated $(R=0.776, p=0.014)$ with the recovered HSVd transcripts (Fig. 1D). HSVd transcripts and $v d-s R N A s$ represented (in average) respectively, the $0.002 \%$ and $0.047 \%$ of the recovered reads at $10 \mathrm{dpi}$, the $0.024 \%$ and $1.5 \%$ at $17 \mathrm{dpi}$ and the $0.02 \%$ and $2.9 \%$ at $24 \mathrm{dpi}$ (Supplemental Table S3 and S4). Our results support that both, viroid transcripts and vd-sRNAs may be detected in systemic tissues in an irregular manner in early infection phases (10 dpi) and more consistently since the $17 \mathrm{dpi}$.

\section{Modifications in the host siRNA/miRNA population}

Associations between SRNA expression profiles (considering control/infected plants and their biological replicates in the three time points) were evaluated using Principal Components Analysis (PCA). The percentages of variance explained by the first three principal components (PC) were $16.43 \%, 15.01 \%$ and $14.47 \%$, respectively $(45,91 \%$ of the total variance). The biological replicates clustered together (attesting for the reproducibility of our assays) and treatments/time-points groups were significantly $\left(p=3.05 \times 10^{-12}\right)$ separated in the PC space (Figure 2A).

The endogenous cucumber sRNAs exhibited a distribution of read lengths enriched for $24 \mathrm{nt}$ long (34.0\%), followed by 23 nt (23.2\%) and similar accumulations of 25 (14.3\%), 21 (12.0\%) and $22(10.5 \%)$ nt long molecules. Reads of 20 nt represented the least abundant category (6.0\%) (Figure 2B). This difference in accumulation of different sRNA lengths was statistically significant (2-ways non-parametric ANOVA, $p<2.12 \times 10^{-15}, 2.14 \times 10^{-13}$ and $1.30 \times 10^{-15}$ for T1, T2 and T3 respectively). In coincidence with diverse cucumber cultivars $(78,79)$, this effect was predominantly due to the large enrichment in $24 \mathrm{nt}$ long sRNAs in all pairwise comparisons. No 
bioRxiv preprint doi: https://doi.org/10.1101/2022.01.06.475203; this version posted January 6, 2022. The copyright holder for this preprint (which was not certified by peer review) is the author/funder, who has granted bioRxiv a license to display the preprint in perpetuity. It is made available under aCC-BY-ND 4.0 International license.

significant differences were found between HSVd-infected and control conditions regarding the observed global distribution of sRNAs sizes (2-ways non-parametric ANOVA, $p=0.93 ; 0.85$ and 0.55 for $\mathrm{T} 1, \mathrm{~T} 2$ and $\mathrm{T} 3$ respectively).

A

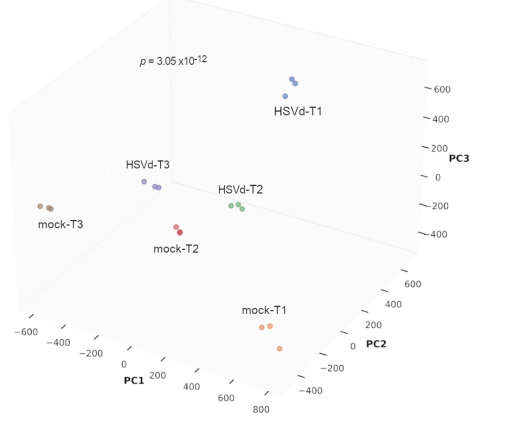

D

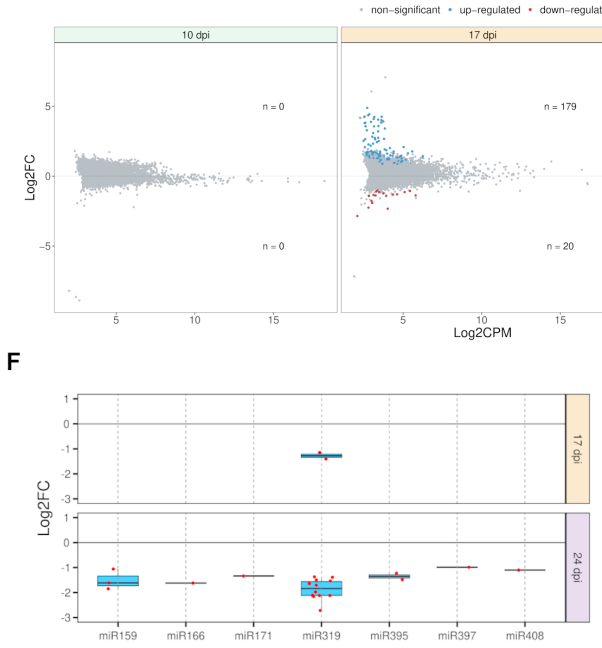

$B$

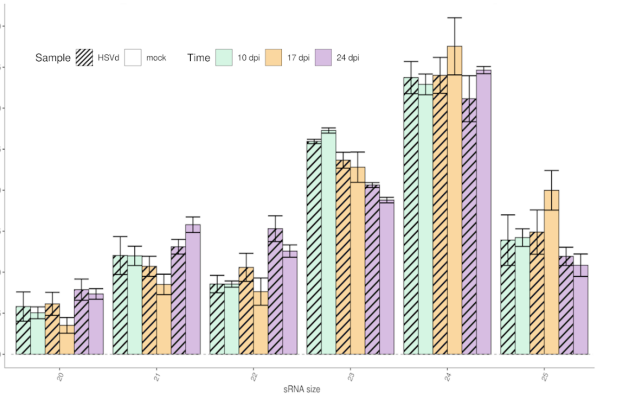

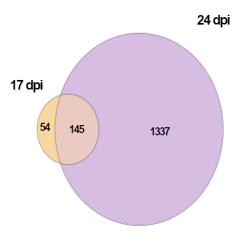

G

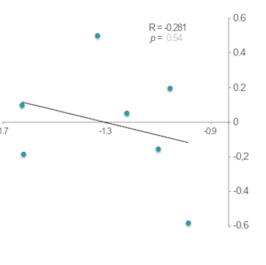

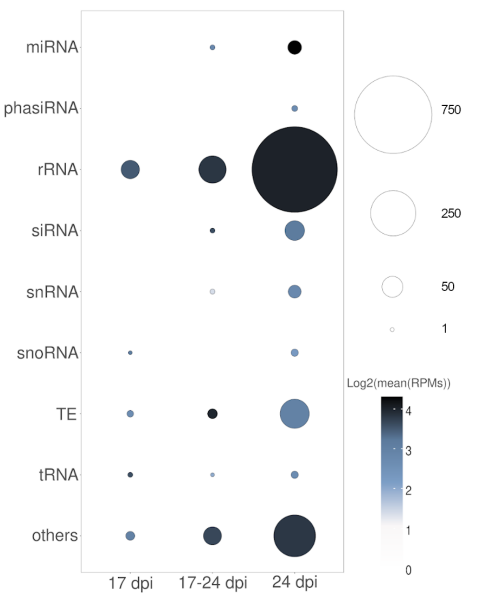

Figure 2: Analysis of the SRNA population recovered from the analyzed cucumber libraries. A) Principal component analysis based on sRNAs accumulation in biological replicates of HSVd-infected and mock-inoculated plants at the three analyzed time-points. The statistical significance was estimated by Mann-Whitney-Wilcoxon test, considering the inter- and intra-group Euclidean distances. B) Diagram showing the means of the temporal relative accumulation and distribution of the total SRNAs reads ranging between 20 and $25 \mathrm{nts}$ (Error bars indicate the SE between biological replicates). The three analized times are represented by colors (green: $10 \mathrm{dpi}$, orange $17 \mathrm{dpi}$ and magenta: $24 \mathrm{dpi}$. Smooth and striped bars represent data arising from mock-inoculated and HSVd-infected plants. C) Venn diagram representing the number of sRNAs responsive to HSVd infection at 17 and 24 dpi. D) from Volcano plot representing the endogenous cucumber SRNAs with significant differential expression in infected plants at the three analyzed time points Blue and red dots indicate up- and down- regulated sRNAs, respectively. The number of differential sRNAs in each time point is detailed. E) Categorization of HSVd-responsive sRNAs. The ball size represents the number of unique reads recovered. The color intensity indicates the sRNA accumulation estimated by the Log2 of the mean of the normalized reads. F) Box-plot analysis showing the general expression value observed for each miRNA-family member (dots) at 17 and $24 \mathrm{dpi}$. The differential expression of each miRNA family is represented by the median (internal box line) of the LFC values. G) Scatter plot showing the non-significant negative correlation (estimated by Pearson correlation coefficient) between the expression levels of the seven miRNAs responsive to HSVd infection and the accumulation of their targets in infected plants.

Only a reduced number (1536 unique reads) of endogenous sRNAs showed significant altered accumulation in HSVd-infected plants at 17 and 24 dpi (no differential sRNAs were detected at 10 dpi) (Fig. 2C). 145 sRNAs were differentially expressed at both 17 (T2) and 24 (T3) dpi, while 54 and 1337 endogenous sRNAs, showed specific differential accumulation at T2 and T3, respectively. A total of 1246 (81,12\%) and 290 (18.88\%) sRNAs increased or decreased, respectively, their accumulation in response to infection. Under a temporal viewpoint, although no differentially expressed sRNAs were detected at $10 \mathrm{dpi}$ (T1), 199 reactive sRNAs (179 overexpressed and 20 down-regulated) were identified at $17 \mathrm{dpi}$ (T2) (Fig. 2D). The most evident alteration in the accumulation of endogenous sRNAs was observed at 24 dpi (T3) in which 1482 
reads (1202 up-regulated and 280 down-regulated) were identified as differentially expressed in HSVd-infected plants. The 68.42\% (1051 reads) of the differential sRNAs were categorized as derived from ribosomal RNA (r-sRNAs), while the $7.55 \%$ (116 reads) from Transposable Elements (TE-sRNAs), and the $3.06 \%$ corresponded to 47 reads identified as small interfering RNAs (siRNAs) previously described in cucumber (Fig. 2E). A minor representation (1.5\%) was observed for known microRNAs (miRNAs), sRNAs derived from snRNAs (1.3\%), tRNAs (0.52\%), snoRNAs $(0.39 \%)$ and phased siRNAs $(0.2 \%)$. The $17.06 \%$ of the sRNAs with differential expression were derived from unidentified or not representative functional categories.

We identified seven miRNA families (miR158, miR166, miR171, miR319, miR395, miR397 and miR408) with differential expression associated to HSVd-infection (Fig. 2F). Except for miR319 (with 14 different related sequences), reactive families were represented by one (miR166, miR171, miR397 and miR408), two (miR395) or three (miR159) unique sequences. All members in each miRNA family decreased in a coordinated manner in response to HSVd-infection (Supplemental Table S5). The miR319 was the unique family that exhibit differentially expressed members at 17 (T2) and 24 (T3) dpi, the rest of the reactive miRNAs were only differential at 24 dpi. The differential expression values of the reactive miRNAs were low (in any case LFC > -1,6), suggesting that the regulatory pathways mediated by miRNAs are not particularly affected by viroid infection. The observation that such miRNAs negatively correlated in a non-significant manner $(\mathrm{R}=-0.281, p=0.541)$ with their target transcripts reinforced this notion (Fig. $2 \mathrm{G}$ ).

\section{Biogenesis and functional role of HSVd-derived SRNAs}

A total of $1,523,822$ reads (representing 7,428 unique sequences) fulfilling the conditions (detailed in material and methods) required to be unequivocally considered as HSVd-derived sRNAs (vd-sRNAs) were recovered from the three analyzed time points (Supplemental Table S3). Analysis of polarity distribution revealed a slight but significant $\left(p=2.2 \times 10^{-16}\right)$ difference according to the strand of origin of the vd-sRNAs (58.1\% sense and $41.9 \%$ antisense) (Figure 3A). This polarity proportion was maintained constant in the three analyzed time points (Supplemental Figure S2). Considering each sRNA size-class individually, the sense-strand derived sRNAs were the predominant, except in $20 \mathrm{nt}$ and $23 \mathrm{nt}$ in length vd-sRNAs (Figure 3B). In contrast, a comparable accumulation rate for both plus and minus reads was observed when unique vd-sRNAs were considered (Figure 3C). Vd-sRNAs were mainly of 24 nt (68.9\%, 57.1\% and 49.0\%,) and $21 \mathrm{nt}(15.5 \%, 24.1 \%$ and 30.0\%), for T1 (10dpi), T2 (17 dpi) and T3 (24 dpi), respectively. Except for 22 nt (10.3\%, 11.8\% and 13.2\%), vd-sRNAs of 20, 23 and 25 nt accumulated under $5 \%$ of the total sRNAs homologous to HSVd in the three analyzed time points (Figure 3D). Regarding predominant vd-sRNAs (24 nt and $21 \mathrm{nt}$ ) was evident that while 
the proportion of 21 nts vd-sRNAs increases during viroid-infection, the accumulation of $24 \mathrm{nt}$ reads recovered from infected plants decreases during the analyzed period. The observation that a comparable size-evolution pattern was not detected for endogenous sRNAs (Supplemental Figure S3), supports that this significant (2-ways non-parametric ANCOVA, $p<$ 0.031 and 0.039 , for 21 and 24 nt respectively) temporal bias in the accumulation of this type of vd-sRNAs is specific for the HSVd-RNA and not related to the cucumber sRNAs biogenesis pathway. Categorized according to their 5- terminal nucleotide, vd-sRNAs with a C-end were the most abundant $(43,8 \%)$, followed by reads with T- $(24,6), G-(17,7)$ and A- $(13,9) 5^{\prime}$ ends (Figure 3E). Similar distribution of 5-ends was observed when total vd-sRNAs were analyzed considering size and temporal evolution (Figure 3F).

When total vd-sRNAs recovered at the three time points were plotted onto HSVd-genome we observed that in general, sense and antisense vd-sRNAs spreading along the entire HSVd genome showed a heterogeneous distribution pattern with four evident hyper accumulation peaks in the position 130 and 254 and -78 and -184, in genomic and anti-genomic HSVd-RNA, respectively (Figure 3G). The hyper accumulation peaks corresponded predominantly to $24 \mathrm{nt}$ sRNAs (Figure $3 \mathrm{H}$ ). This global profile was maintained during the analyzed infection period (Supplemental Figure S4). In contrast to hyper accumulation peaks, we observed that a welldefined region of the viroid-genome comprised between the positions 11 to 38 (sense reads) and 31 to 58 (antisense reads) showed a null or much lower accumulation of vd-sRNAs in the three analyzed time points (Supplemental Figure S5).

It has been previously demonstrated that certain vd-sRNAs can trigger the silencing of endogenous transcripts in infected plants $(21,23,28)$. Although some mRNAs have been proposed to be targeted by HSVd-derived sRNAs, the validation of this activity is still lacking for this viroid (80). Therefore, we performed a degradome analysis starting from the RNA samples obtained (by triplicated) from the mock- and HSVd-inoculated plants at $24 \mathrm{dpi}$. According to our established parameters (Supplemental Figure S6) we found three vd-sRNAs predicted to target three cucumber transcripts with potential cleavage-site identified by degradome analysis and significant decreased expression in infected plants at 24 dpi suitable to be regulated by vdsRNAs via RNA-silencing mechanisms (Fig. 31 and Supplemental Figure S6). 
bioRxiv preprint doi: https://doi.org/10.1101/2022.01.06.475203; this version posted January 6, 2022. The copyright holder for this preprint (which was not certified by peer review) is the author/funder, who has granted bioRxiv a license to display the preprint in perpetuity. It is made available under aCC-BY-ND 4.0 International license.

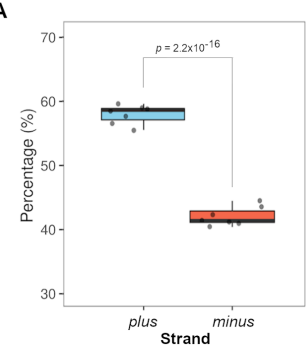

D

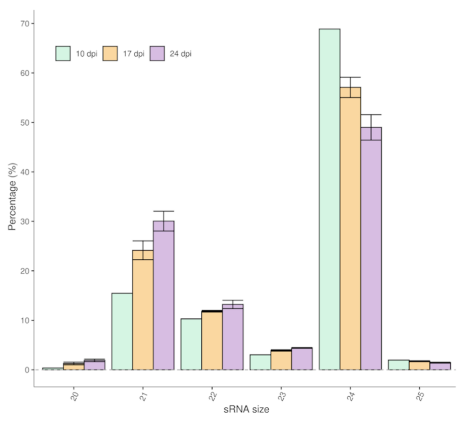

G

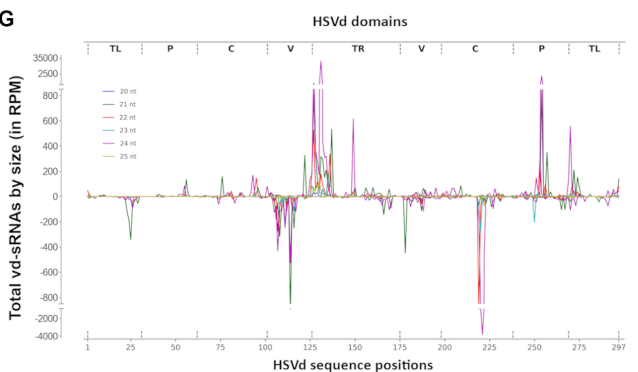

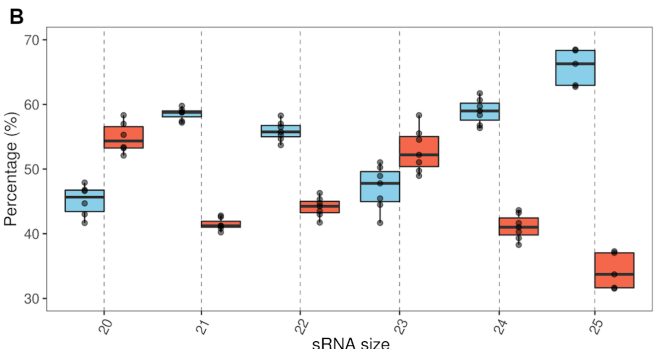

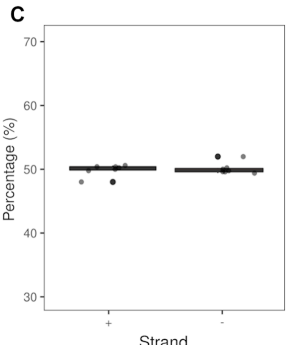

E

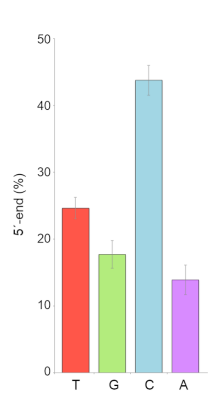

$\mathbf{F}$

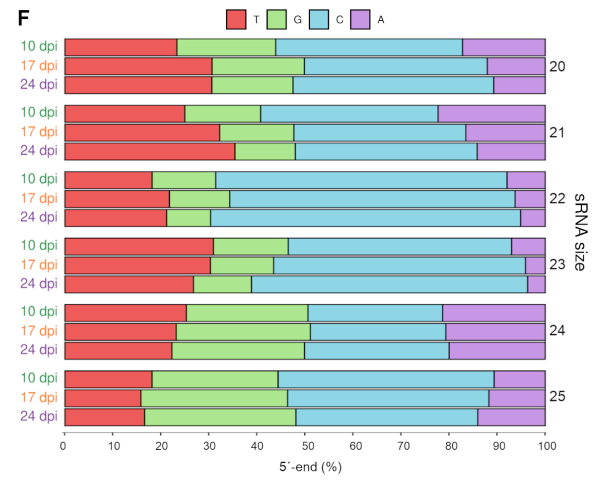

H

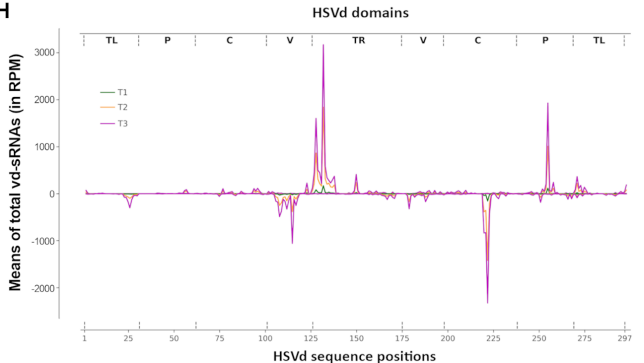

$\mathbf{J}$
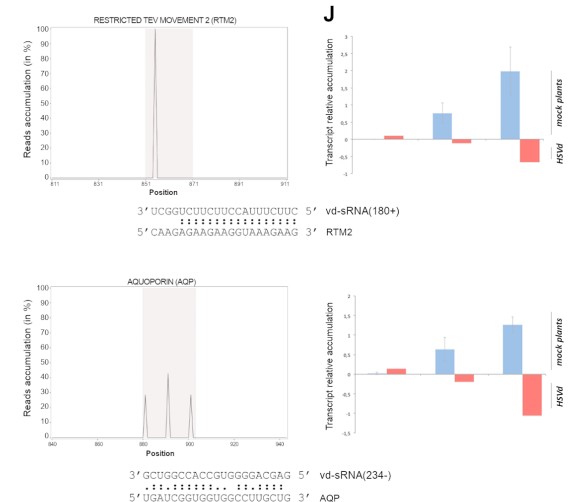

K
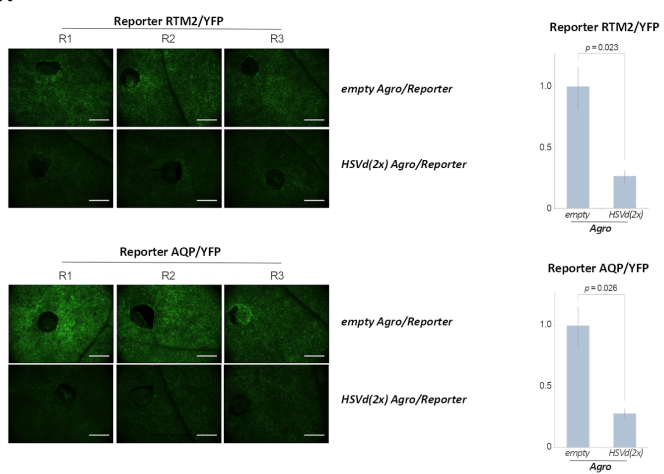

Figure 3: Characterization of vd-sRNAs. A) Box plot analysis of the relative accumulation of the total vd-sRNAs (20 to $25 \mathrm{nt}$ ) derived from genomic (plus) and antigenomic (minus) HSVd-RNA strand at the three analyzed time points. B) Distribution of polarity of the total vd-sRNAs discriminated by size. C) Comparative analysis of unique reads of vd-sRNAs. D) Diagram showing the means of the temporal relative accumulation and distribution of the vd-sRNAs reads ranging between 20 and $25 \mathrm{nts}$ (the error bars indicate the SE). The three analized times are represented by colors (green: $10 \mathrm{dpi}$, orange: $17 \mathrm{dpi}$ and magenta: 24 dpi). E) Graphic representation of the proportion of the 5'- ends in the total recovered vd-sRNAs. The means of the total reads are showed. F) Distribution of the $5^{\prime}$ - ends discriminated by size and infection time. G) Genome view of the vd-sRNAs recovered from infected cucumber plants. The vdSRNAs were plotted according to the position of their 5 '-end onto the HSVd- RNA sequence in either sense (above the $x$-axis) or antisense (below the $x$-axis) configuration. The values on the $y$-axis represent the abundance of total vd-sRNAs (sizes indicated by colors) in the three analyzed time point. H) configuration. The values on the $y$-axis represent the abundance of total vd-sRNAs (sizes indicated by colors) in the three analyzed time point. $\mathrm{H}$ ) Representation of the temporal accumulation of total vd-sRNAs (means of total vd-sRNAs are represented). Vd-sRNAs recovered in each of the three analized
times are represented by colors (green: $10 \mathrm{dpi}$, orange: $17 \mathrm{dpi}$ and magenta: $24 \mathrm{dpi}$ ). I) Representation of the vd-sRNA-cleaved transcripts (RTM2 and AQP, times are represented by colors (green: $10 \mathrm{dpi}$, orange: $17 \mathrm{dpi}$ and magenta: $24 \mathrm{dpi})$. I) Representation of the vd-sRNA-cleaved transcripts (RTM2 and AQP,
upper and lower panel respectively) differentially detected by degradome assay in infected plants. Degradome sequences were plotted onto the predicted targets transcripts. The grey zone indicates the predicted vd-sRNA recognition site (detailed below). The values on the Y-axis represent the relative accumulation of the reads matching onto the $160 \mathrm{nt}$ in length analized region. J) Means of the temporal relative accumulation estimated by sequencing of the target transcripts in infected and control plants (the fine lines indicate the SE). K) Down regulation of the predicted targets mediated by HSVd was validated by transient expression of specific reporters carrying YFP transcriptionally fussed to the RTM2 (upper panel) and AQP (lower panel) target sequences. Reporter expression was determined by fluorescence emission (left) and transcript quantification by RT-qPCR (right). Error bars indicate the SE between biological replicates.

The vd-sRNAs identified as vd-sRNA (180+), vd-sRNA (185-) and vd-sRNA (234-) were 21 nts in length and derived from plus (180+) and minus (185- and 234-) HSVd strands. The potential 
targets for vd-sRNAs were predicted to encode cucumber homologous to RESTRICTED TEV MOVEMENT 2 (RTM2), CELLULOSE SYNTHASE (CesA) and AQUAPORIN (AQP). The relative accumulation of these predicted target-transcripts was consistently decreased through the infection period in contrast to the three vd-sRNA that increase their accumulation over time (Fig. 31 and Supplemental Figure S7), reinforcing the data obtained by degradome analysis. An additional assay performed in $N$. benthamiana plants accumulating HSVd was used to validate the viroid-mediated down regulation of RTM2 and AQP by analysing the fluoresce emission and transcript accumulation of chimeric reporters containing Yellow Fluorescent Protein (YFP) transcriptionally fussed to the transcript sequences predicted to be targeted by vd-sRNAs (180+) and (234-), respectively (Fig. $3 \mathrm{H}$ and I).

HSVd-infection modulates the expression and alternative splicing of hosttranscripts

It is expected that the host transcriptome may change in response to the viroid infection. To test that assumption, RNA extracted from control and infected plants was subjected to RNA-seq. A high proportion (98.33\%) of the recovered transcripts in both infected and control plants mapped to the cucumber genome (Supplemental Table S3), allowing the detection of differentially expressed transcripts (DETs) in all time points. The residual reads corresponded to unassigned transcripts (1.66\%) and HSVd-derived sequences (0.01\%). Considering only reads fully homologous to previously annotated cucumber RNAs, we identified 1125, 515 and 1390 transcripts with significant (FDR $\leq$ 0.05) differential accumulation in HSVdinfected plants, at 10, 17 and 24 dpi, respectively (Fig. 4A and Supplemental Table S6). A total of 925 (at $10 \mathrm{dpi}$ ), 288 (at $17 \mathrm{dpi}$ ) and 1052 (at $24 \mathrm{dpi}$ ) host-transcripts presented time-specific differential accumulation (Fig. 4B). Considering LogFC $\leq-1$ or $\geq 1$ as cut-off value, the list of differential transcripts was reduced to 10 up-regulated at T1, 55 (20 up-regulated and 35 downregulated) at T2 and 271 (46 up-regulated and 225 down-regulated) at T3 (size-increased dots in Fig. 4A). The 10 up-regulated genes recovered at $10 \mathrm{dpi}$ were time-specific, whereas 27 and 243 transcripts showed specific differential expression at 17 and 24 dpi, respectively (Fig. 4C). Under a temporal viewing, it was obvious that the host transcripts with increased accumulation were predominantly (695 transcripts, 61.7\%) recovered at $10 \mathrm{dpi}$, while down-regulation was predominant at $17 \mathrm{dpi}$ (302 transcripts, 58.64\%) and 24 dpi (975 transcripts, 70.14\%). A similar trend was observed when expression values with a LogFC $\leq-1$ or $\geq 1$ were considered. The amplitude (estimated by the variance of the differential expression values) and diversity (estimated by the number of DETs) of the transcriptional response to HSVd was clearly increased during the analyzed period of infection (Fig. 4D). 
bioRxiv preprint doi: https://doi.org/10.1101/2022.01.06.475203; this version posted January 6, 2022. The copyright holder for this preprint (which was not certified by peer review) is the author/funder, who has granted bioRxiv a license to display the preprint in perpetuity. It is made available under aCC-BY-ND 4.0 International license.

A

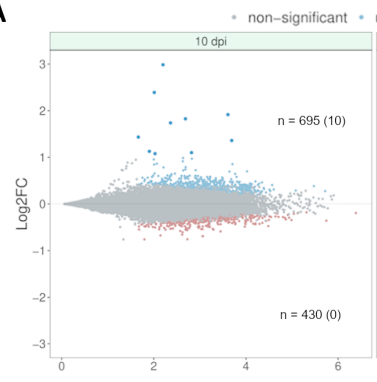

D

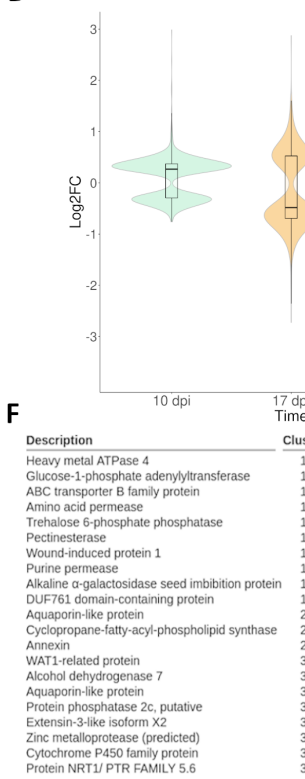

I

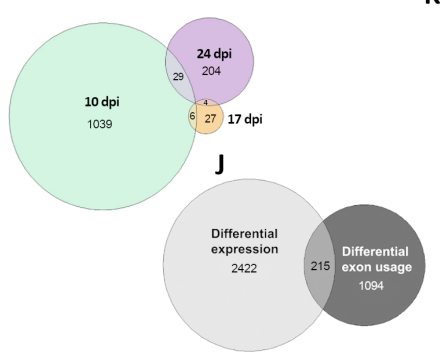

K

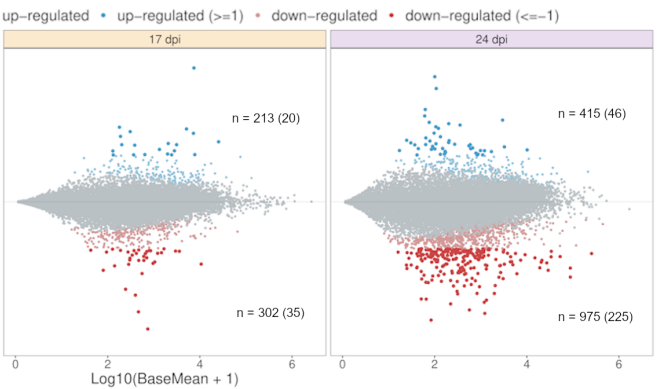

E
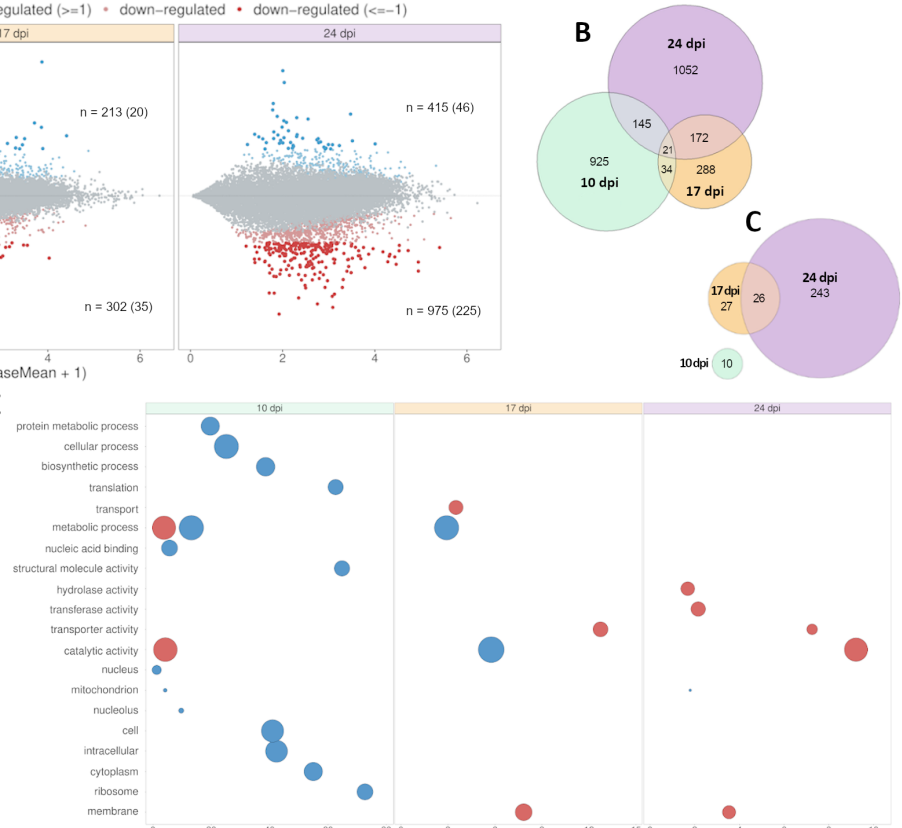

$\circ$

H
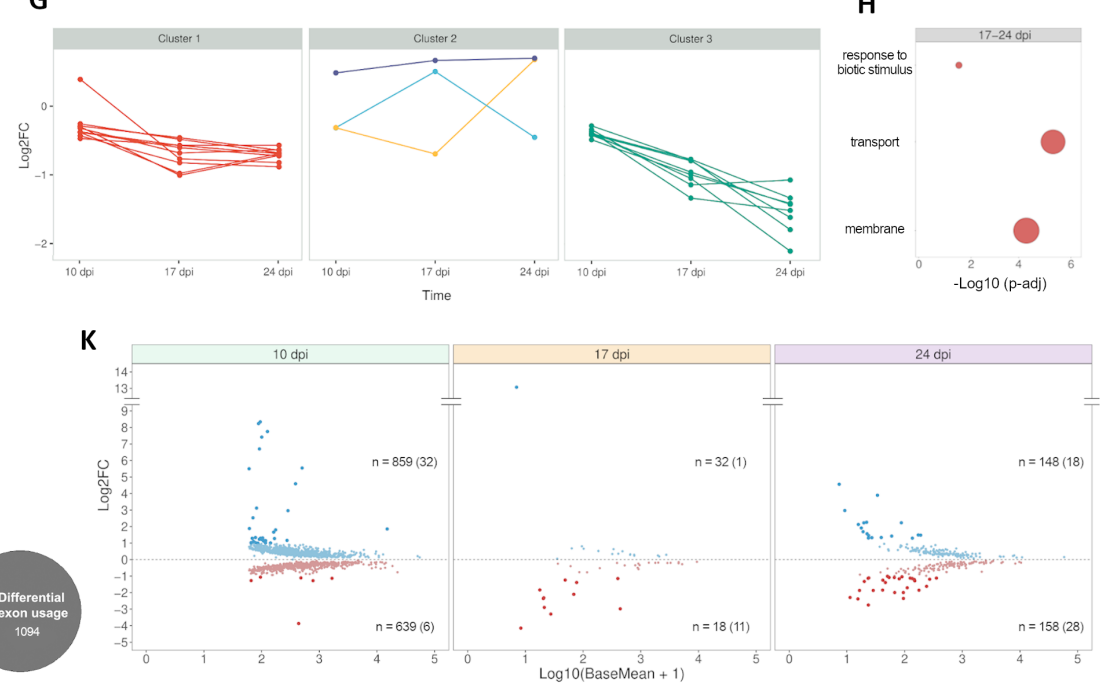

Figure 4: Host-transcriptional alterations associated to HSVd-infection. A) Volcano plot representing the differentially expressed transcripts (DET) in infected cucumber plants at 10,17 and 24 dpi. Blue and red dots indicate up- and down- regulated transcripts, respectively. DET with LFC $\geq 1$ or $\leq-1$ are represented by bold dots and the number is indicated in brackets. Venn diagram showing all the DET (B) and those with $L F C \geq 1$ or $\leq-1$ (C) identified at 10,17 and $24 \mathrm{dpi}$ D) Violin plot representing the temporal profile of the transcriptional alteration associated to HSVd-infection. Internal box line indicates the median of the LFC values. E) Gene ontology analysis (plant GOSlim) for DETs identified in HSVd-infected plants at 10,17 and $24 \mathrm{dpi}$. Circle size represents level of enrichment and color under- (red) or up-regulated (blue) response. The - $\log (10)$ of the adjusted $P$ values is represented in the X-axis. F) Detail of the 21 transcripts differentially expressed at the three analyzed time-points. G) Clustering analysis of time-course expression profiling of transcripts with differential expression at 10, 17 and 24 dpi. H) Gene ontology analysis (plant GOSlim) for transcripts differentially expressed at both 17 and $24 \mathrm{dpi}$ (LFC $\geq 1$ or $\leq-1)$. I) Venn diagram showing the number of DEU events identified at 10,17 and $24 \mathrm{dpi}$ and $(\mathrm{J})$ transcripts showing common and specific regulation by differential expression or exon usage. K) Volcano plot representing the events of differential exon usage (DEU) in infected plants at 10,17 and 24 dpi. Blue and red dots indicate up- and down- regulated exons, respectively. Events of DEU with LFC $\geq 1$ or $\leq-1$ are represented by bold dots and the number is indicated in brackets.

According to the analysis of GO-terms, transcriptional response to HSVd-infection was characterized by a significant enrichment in genes associated to diverse biological categories mainly related to metabolic and cellular process, catalytic activity, ribosome metabolism, cellular/intracellular components, transport and membrane (Fig. 4E and Supplemental Table S6). Under a global viewpoint, was evident that the wide-range of enriched functional categories observed at 10 dpi (15 categories), was decreasing during infection (6 enriched- 
categories at $24 \mathrm{dpi}$ ). Searching for host genes potentially involved in a more persistent response to HSVd-infection we identified cucumber transcripts differentially expressed during the three infection phases. A total of 21 transcripts were identified as significantly responsive to infection at 10, 17 and $24 \mathrm{dpi}$ (Fig. 4F and Supplemental Table S7). Except for three specific transcripts up-regulated in at least one of the analyzed time points (Fig. 4G, cluster 2), the common response of the other 18 differential transcripts to HSVd-infection was a stable (cluster 1) or temporally increased (cluster 3) down regulation. Genes included in this common response were functionally related to transport, membrane activity and response to biotic stimulus. This functional specialization was also supported by GO-terms enrichment when were considered only transcripts with LFC $\geq 1$ or $\leq-1$ with common differential expression at late (17 and $24 \mathrm{dpi}$ ) infection phases (Fig. 4H). Differential transcripts without previous annotation (and consequently not considered in our study) were analyzed as is described in Methods section (Supplemental Figure S8). The obtained results revealed that such transcripts (mainly identified as non-coding RNAs) exhibited minimal alterations in response to HSVd infection (Table S8).

Alternative splicing (AS) has been proposed as a regulatory mechanism crucial for the modulation of the plant development and response to virus infection (81). Sequenced transcripts were analyzed with DEXSeq tool to infer the AS landscapes of cucumber plants and determine their differential patterns during HSVd infection. We identified 1589 differential exons derived from 1309 intron-containing multiexonic genes that were alternatively spliced in infected plants (Fig. 4I). Among such transcripts, only 215 (5.76\%) also showed differential expression (Fig. 4J). Under a global viewpoint, significant differential exon usage (DEU) was predominantly observed in infected plants at 10 dpi (1498 exons with DEU), less frequent at 24 dpi (306) and a residual phenomenon at 17 dpi (with 50 alternative splicing events identified) (Fig. 4K and Supplemental Table S9). Differential exon usage in response to HSVd-infection was comparable at early (38 exons) and late infection phases (46 exons) when we considered only LogFC values $\geq 1$ or $\leq-1$ (size increased dots in Fig. 4K). Regarding the temporal trend in the differential exons usage, our results support that HSVd infection is associated to a predominant retention of exons at 10 dpi showing 32 over-represented exons with LogFC values $\geq 1$ (Fig. 4K). In contrast, the underrepresented exons (with LogFC $\leq-1$ ) were prevalent at 17 (11 exons) and 24 dpi (28 exons). Transcripts exhibiting frequent DEU, at both early (10 dpi) and late (24 dpi) infection phases were predominantly involved in primary metabolism-related process (Supplemental Figure S9). Additionally, transcripts associated to membrane and transport, were enriched at $10 \mathrm{dpi}$. 


\section{Epigenetics alterations associated to HSVd-infection}

To analyze if HSVd-infection modifies the epigenetic landscape of cucumber plants, wholegenome bisulfite sequencing (WGBS) libraries were constructed. Global analysis (considering the total percentage of cytosine methylation in $\mathrm{CG}, \mathrm{CHG}$ and $\mathrm{CHH}$ sequence context) revealed a non-significant hypomethylation in infected plants at $10 \mathrm{dpi}(70.39 \%$ and $67.21 \%$, for control and infected respectively), followed by a slight hypermethylation at $17 \mathrm{dpi}(75.04 \%$ and $76.93 \%)$ that was significant (70.6\% and $73.21 \%$ for control and infected plants, respectively) at $24 \mathrm{dpi}$ (Fig. 5A, upper panel). These differences were maintained when we considered total methylation level for each sequence context specifically (Fig. 5A, lower panel). A similar scenario was observed when the total methylation levels were analyzed, considering each cucumber chromosome individually (Fig. 5B). To obtain a more precise picture of the specific methylation changes, we used DMRcaller to identify differentially methylated regions (DMRs) for the three $\mathrm{CG}, \mathrm{CHG}$, and $\mathrm{CHH}$ sequence contexts. Only significant DMRs with differences in methylation $\geq 15 \%$ were considered. The hypomethylated CG DMRs were the most abundant during the analyzed infection period. In contrast hypermethylated DMRs were most abundant considering CHG and $\mathrm{CHH}$ sequence context (Fig. 5C).

This global trend was maintained constant during the three analyzed time points. Considering the intensity (assumed as the median of the DMRs values) of the methylation alterations in each time and context, it was evident that the higher differential values for both hypo (18.4\%, 18.0\% and $18.8 \%$ for T1, T2 and T3 respectively) and hypermethylation (19.8\%, 17.5\% and 18.3\% for T1, T2 and T3 respectively) were observed for the GHG context, while a lower number of alterations was observed for the $\mathrm{CHH}$ DMRs (11.3\%, 9.0\% and 10.8\%) for loss of methylation at T1, T2 and T3 respectively and $12.5 \%, 9.9 \%$ and $11.6 \%$ for gaining in identical time points (Fig. 5D). For the DMRs of the CG context, the medians of the differential alterations were near to the $15 \%$ during the entire analyzed period. Considering the functional categories of the genomic position of the DMRs, approximately $60 \%$ of the observed CG DMRs in the three analyzed time points were mapped in regions predicted as coding sequences (CDS) and introns, while intronic regions constituted the predominant DMRs at the CHG context (Fig. 5E). As expected, during the analyzed period, $\mathrm{CHH}$ DMRs were enriched in TEs-associated regions and strongly diminished in CDS.

It has been previously demonstrated that HSVd infection promotes alterations in the epigenetic landscape of regulatory regions of the ribosomal DNA (rDNA) (82) to reconfigure a functional scenario that encourages its replication in cucumber plants (48). To extend this study, we analyzed if rDNA was specifically identified as a significant DMR. A region of 150 nt comprised 
bioRxiv preprint doi: https://doi.org/10.1101/2022.01.06.475203; this version posted January 6, 2022. The copyright holder for this preprint (which was not certified by peer review) is the author/funder, who has granted bioRxiv a license to display the preprint in perpetuity. It is made available under aCC-BY-ND 4.0 International license.

between positions 1984 - 2133 of the ribosomal genes and overlapping with their promoter region was identified as hypomethylated DMR in CG and CHG contexts at 10 dpi (boxed region in Fig. 5F and Supplemental Figure S9). Moreover, considering the total methylation levels at CG context, it was evident that HSVd induces a significant $\left(p=2.2 \times 10^{-16}\right)$ hypomethylation of the rDNA (79.57 and 73.75 of total methylation for mock and HSVd-infected plants, respectively) at 10 dpi.
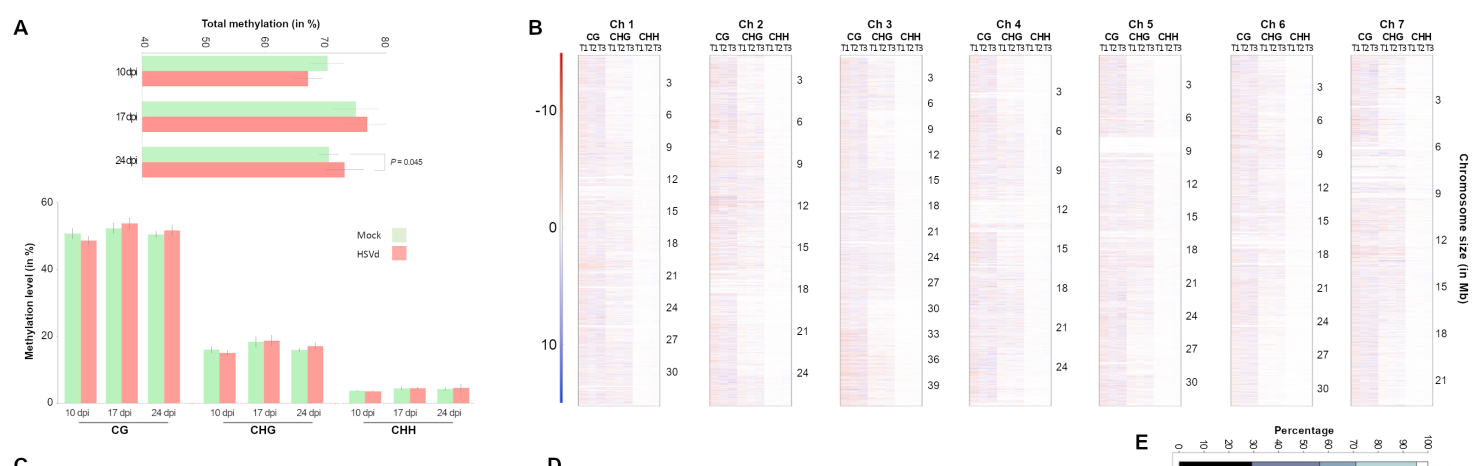

C
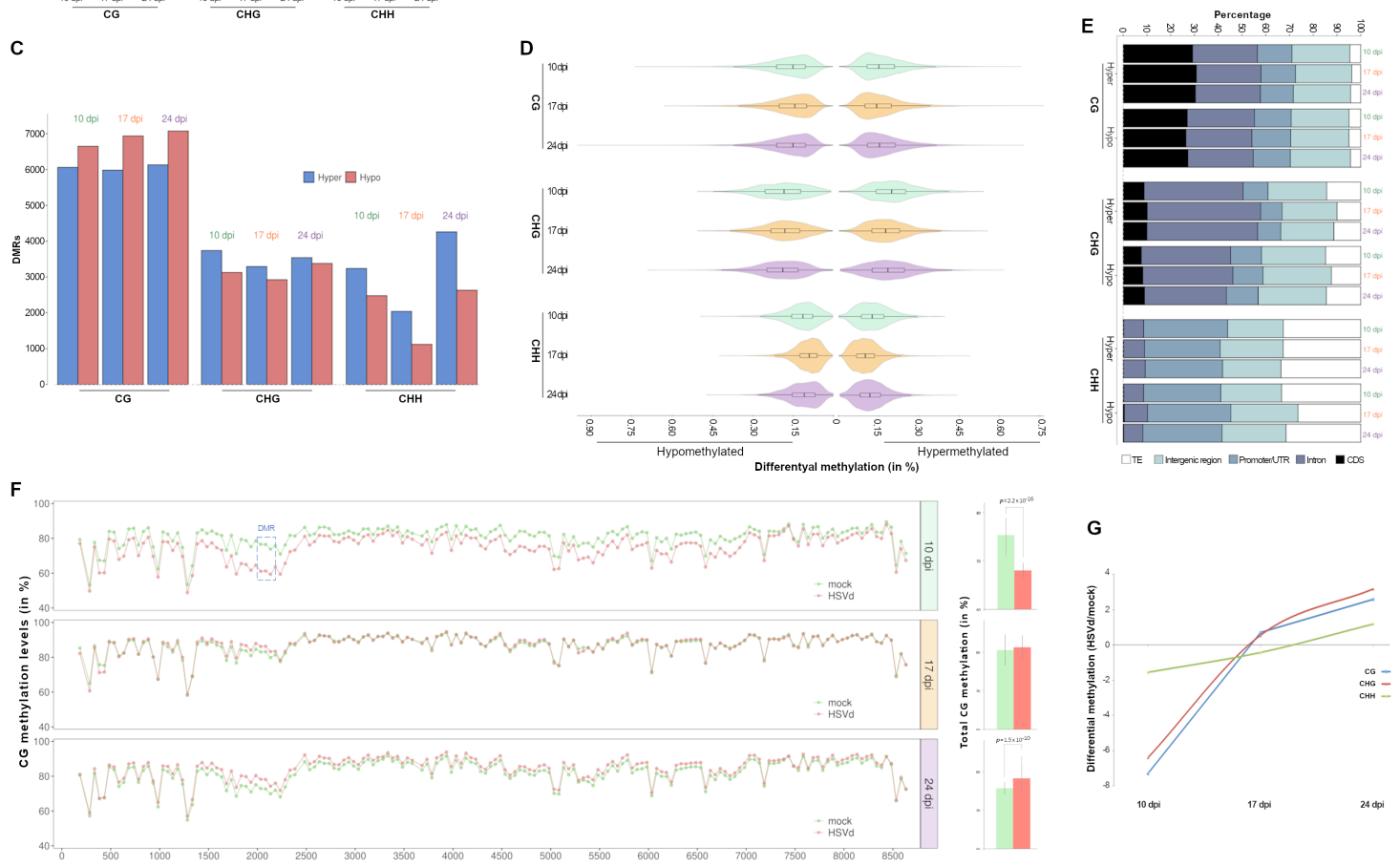

G

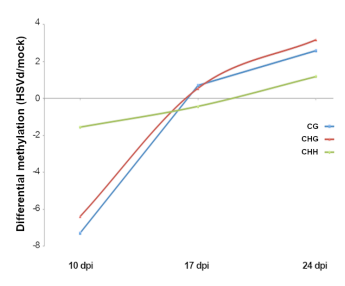

Figure 5: HSVd induces alterations in the cucumber epigenetic landscape. A) Graphic representation of the total cytosine methylation mean values of HSVd-infected and control plants at 10, 17 and $24 \mathrm{dpi}$ (upper panel) and discriminated by sequence context (lower panel). Error bars indicate the standard error between replicates. B) Chromosome view of the temporal evolution of the differential methylation profiles in HSVd-infected plants. Red and blue lines represent hypomethylated and hypermethylated regions, respectively. C) Number of significant hyper- or hypo- differentially methylated regions (DMRs) in the three sequence contexts (CG, CHG and CHH) identified in infected plants at 10,17 and 24 dpi. D) Violin plot representing the temporal profile of the epigenetic alterations (estimated by significant DMRs) associated to HSVd-infection. Internal box line identified in infected plants. F) Global view of the methylation profiles in CG context in ribosomal RNA transcriptional unit at 10,17 and 24 dpi in HSVd-infected (red) and control (green) plants. In the left part each dot represents the mean methylation values of 50 nucleotides while in the right the total mean methylation values of the whole ribosomal unit are represented. A Significantly hypomethylated DMR identified in infected plants at $10 \mathrm{dpi}$ and matching to promoter region is highlighted. G) View of the temporal evolution of the differential methylation in the three sequence contexts ( $\mathrm{CG}, \mathrm{CHG}$ and $\mathrm{CHH}$ ) observed in ribosomal RNA transcriptional unit during infection.

Although the downregulation of the methylation levels was more evident in the promoter region (identified as a significant DMR), the hypomethylated status was extended to the entire ribosomal gene (Fig. 5F - upper panel). Epigenetic alteration of the rDNA associated to HSVd infection was revealed as a dynamic phenomenon, being comparable to mock at 17 dpi (Fig. 5F - central panel) and significantly $\left(1.5 \times 10^{-10}\right)$ hypermethylated at $24 \mathrm{dpi}$ with total-CG 
methylation levels of $80.94 \%$ and $83.37 \%$ for mock and HSVd-infected plants, respectively (Fig. 5F - lower panel). This dynamic alteration of the host epigenetic landscape was also observed when $\mathrm{CHG}$ and $\mathrm{CHH}$ sequence contexts were considered (Fig. 5G and Supplemental Figure S10). Under a temporal viewpoint, we noted that while the significant hypomethylation of ribosomal genes was coincident with the phase of increasing HSVd accumulation, the recovery of the hypermethylation status in host rDNA was in parallel with the stabilization (or slight decrease) of the HSVd transcripts in infected plants (Supplemental Figure S11).

Interplay between methylation and transcriptional regulation in infected plants To analyze if the changes in the methylation level observed in HSVd-infected plants could be associated to alterations of their transcriptional activity we integrated the data obtained by RNA-seq and WGBS assays. 113 protein-coding transcripts with strong (LogFC $\leq-1$ or $\geq 1$ ) differential expression at $24 \mathrm{dpi}$ containing at least one DMR in any of these regions (promoter/UTR, CDS and intron), were selected for this analysis (Supplemental Table S10). We observed that 68 of these 113 genes - representing a significant ( $p=0.038$ in Exact binomial test) proportion (60.2\%) of the analyzed transcripts - were characterized by containing at least one antagonistic DMR (hypomethylated for over accumulating transcripts or hypermethylated for downregulated transcripts) (Supplemental Table S11). Interestingly, a significant negative correlation was observed when we compared their expression levels (estimated by LogFC) with their global methylation status (determined by DMR analysis in the three sequence contexts) in promoter/UTR and CDS regions ( $p=0.003$ and 0.014, respectively) (Fig. 6A). No significant correlation was observed for DMRs matching to introns ( $p=0.148$ ). A more detailed analysis (focused on each context separately) evidenced that the negative correlation was mainly associated to DMRs in the CG context (Fig. 6B and Supplemental Figure S12). Next, we compared the expression and methylation levels of these 68 genes at 10, 17 and 24 dpi to obtain a global overview of the temporal evolution of the interplay between cytosine methylation and transcriptional response during HSVd-infection. Our results indicate that while the intensity and the density of the global methylation of the analyzed genes (estimated by the mean values and the total number of the DMRs) were increased during the analyzed period (Fig 6C, upper panel - left), their transcriptional activity (estimated by the mean of the LogFC values and the number of DET) was consistently down-regulated (Fig 6C, lower panel - left). In a similar way, the transcripts overaccumulating during HSVd-infection were associated to a temporally increased hypomethylation status of their respective genes (Fig 6C, right - lower and upper panel respectively). According to their biological function, the cucumber genes showing 
bioRxiv preprint doi: https://doi.org/10.1101/2022.01.06.475203; this version posted January 6, 2022. The copyright holder for this preprint (which was not certified by peer review) is the author/funder, who has granted bioRxiv a license to display the preprint in perpetuity. It is made available under aCC-BY-ND 4.0 International license.

methylation levels antagonistic to transcripts accumulation mainly encoded proteins related to membrane (associated to stress response or transport), oxidation-reduction and metabolic processes (Supplemental Table S11). Additionally, some genes encoding for nucleus components, Transcription Factors and protein involved in protein-protein interactions, were also identified.

A
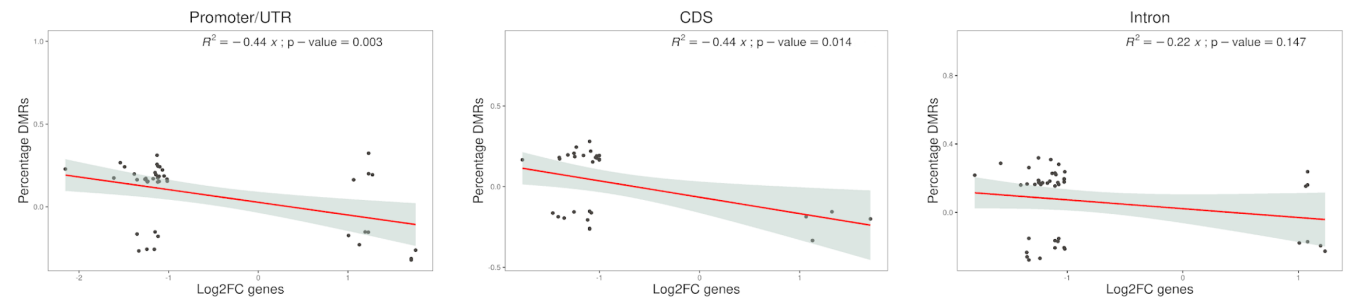

B

$C G$
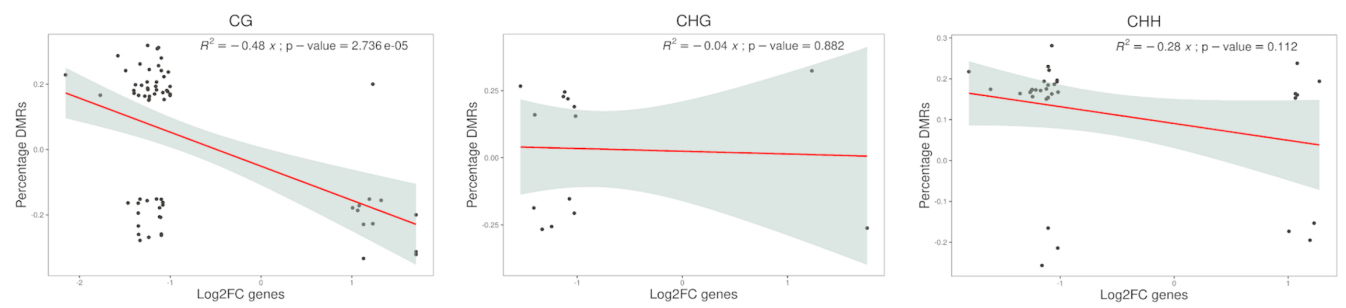

C
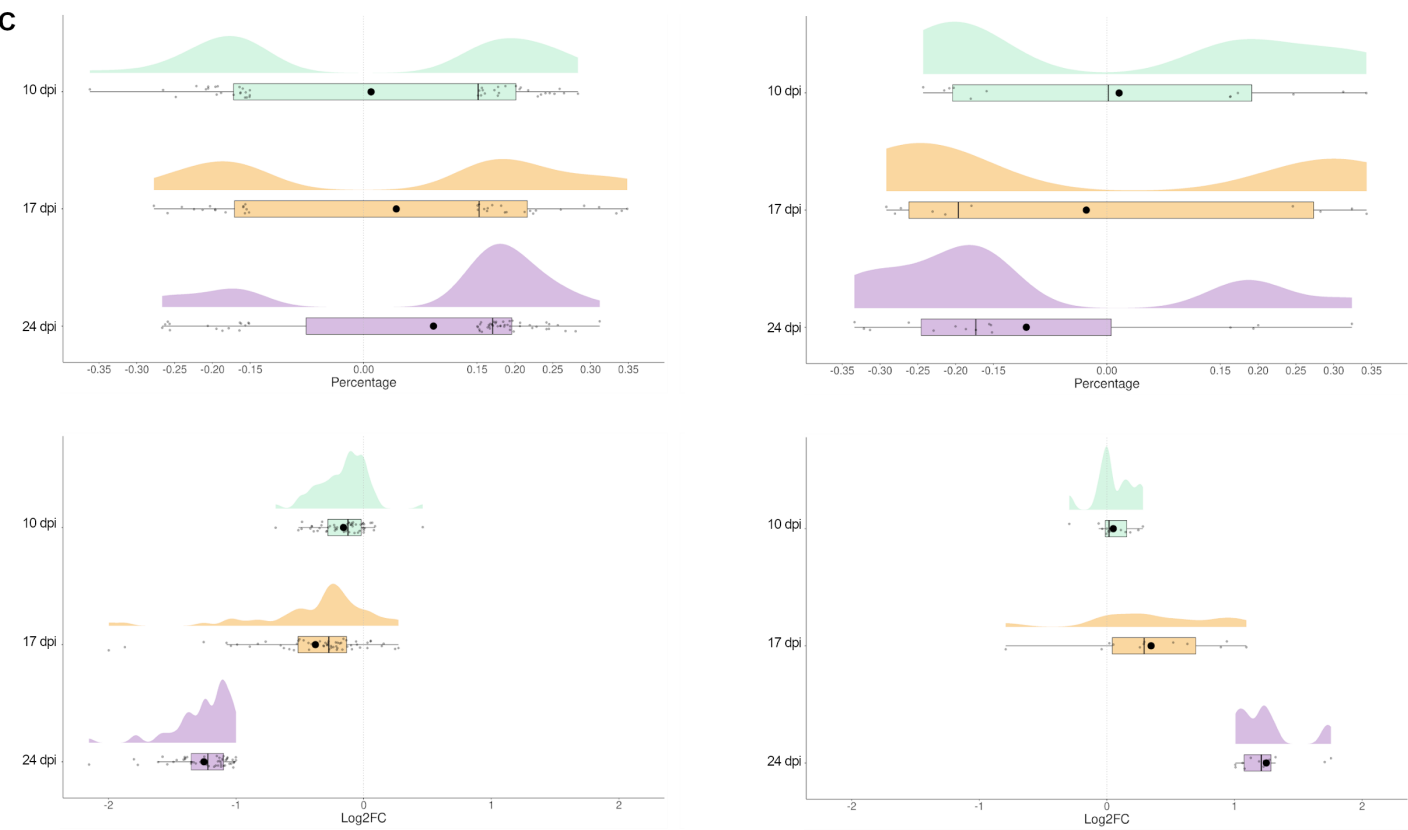

Figure 6: Association between transcriptional alterations and epigenetic changes induced by HSVd-infection. Scatter plots showing the negative correlation (significance estimated by Pearson correlation coefficient) between the expression levels of differential genes containing at least one antagonistic DMR and their global methylation status. A) Considering the three selected gene regions (Promoter/UTR, $\mathrm{CDS}$ and intron) and B) considering methylation sequence context. C) Raincloud plots showing the temporal correspondence between the sense and the intensity of the epigenetic changes (upper panels) and the transcriptional activity (lower panels) in response to HSVd-infection. The temporal increase in the intensity of the hypermethylated (upper left) and hypomethylated (upper right) DMRs represented by the mean of the differential methylation (black dots) is associated with an evident decrease (lower right) or increase (lower left), respectively (in intensity) of the transcriptional activity estimated by the LFC mean values (black dots).

Regarding the regulation of non-coding transcripts by epigenetic changes, we analyzed the transcriptional activity of the cucumber ribosomal genes, which has been earlier described to be modulated by HSVd infection (47). Our data showed that the significant hypomethylation of 
rDNA observed at early infection phases was associated to an increased transcriptional activity of rRNA genes determined by the increased accumulation of primary precursors of the ribosomal transcripts (pre-rRNAs) in infected plants (Supplemental Figure S13, left panel). During rRNA transcription, RNA polymerase I transcribes length-units pre-rRNAs that are extensively processed into $18 \mathrm{~S}, 5.8 \mathrm{~S}$ and $25 \mathrm{~S}$ units by the sequential deletion of external and internal transcribe spacers (ETS and ITS, respectively) (83). Consequently, the differential accumulation of pre-rRNAs was estimated by considering the transcripts matching to ITS1 and ITS2 regions. Finally, the over accumulation in infected plants of rRNA-derived sRNAs (rbSRNAs), an indirect indicator of the transcriptional activation of normally inoperative rRNA transcriptional units (84), provided additional support to the link between rDNA hypomethylation and transcriptional deregulation (Supplemental Figure S13, right panel).

\section{DISCUSSION}

In coincidence with other cellular parasites, viroids are compelled to subvert all sorts of cellular factors and reprogram host gene expression in order to modulate (into their own benefit) complex plant regulatory networks. Assuming this functional scenario, it is expected that the type of affected host-regulatory pathways as well as the intensity of the induced alterations may vary considerably during the infection process. However, our knowledge about the functional diversity and temporal evolution of the global plant response to viroid infection is limited $(3,6)$.

Here, we have addressed this question by means of a temporal analysis of the global response to HSVd infection focussed on three host regulatory levels small RNA interference, modulation of the transcriptional activity and epigenetic modifications. This integrative analysis allowed us to determine that during the analyzed infection period cucumber plants follow different strategies to modulate their cellular homeostasis in response to the functional alterations induced by HSVd replication and accumulation.

The early detection (at $10 \mathrm{dpi}$ ) of viroid-derived transcripts and sRNAs in non-inoculated apical tissues evidenced the capacity of HSVd to develop a relatively quick systemic infection in cucumber plants. However, the observation that viroid transcripts are recovered from only two out the three biological replicates and in a heterogeneous level (27 and 2487 and 2.94 and 1421, normalized reads, for genomic and vd-sRNAs respectively) suggests that initial infection phase is highly variable and maybe dependent on yet unknown specific viroid-host interactions or that minimal variations on the plant phenological stage could condition these early events. 
Analysis of the small RNA data evidenced that although sRNAs derived from plus polarity transcripts were predominantly recovered from infected plants at the three analyzed time points, both plus and minus HSVd replication intermediates are potential substrates for host RNA silencing machinery at different infection phases. Our results are in consonance with previous works (performed at specific infection times) in that the recovery of small RNAs of both polarities supports the predominant involvement of viroid replication intermediates as source of vd-sRNAs in plants infected by both nuclear $(16,17,85-87)$ and chloroplastic $(15,88)$ viroids. Considering size distribution, $24 \mathrm{nt} v d$-sRNAs were the predominant at the three analyzed time points, followed by 21 nt in length sequences. However, it was evident that this size-distribution profile was modified during HSVd infection, being characterized by a temporal increasing of the global proportion of $21 \mathrm{nt}$ that negatively correlates with the accumulation of $24 \mathrm{nt} v \mathrm{~d}$-sRNAs. These results support that the processing/accumulation of $24 \mathrm{nt} v \mathrm{~d}$-sRNAs is predominantly associated to initial infection steps, while the accumulation of the 21 nt class constitutes an event characteristic of well-established infectious processes. The observation that a similar scenario is not observed when cucumber sRNAs were considered evidenced the independence of this phenomenon with the natural evolution of the endogenous sRNAs biogenesis. This temporal trend may explain the inconsistency between our data and previous results supporting that $21 \mathrm{nt}$ in length is the more abundant class of vd-sRNA in HSVd-infected cucumber plants at latest infection phases $(80,86)$. When distribution of vd-sRNAs along HSVdgenome was analyzed we observed that reads matching onto four specific regions, represent approximately the $40 \%$ of the recovered reads in the three analyzed time points. In contrast, two HSVd-RNA regions exhibited null or extremely lower accumulation of vd-sRNAs. The existence of these hyper- and hypo- accumulating regions (detected in both HSVd strands) may be explained assuming the existence of certain viroid-RNA regions exhibiting differential accessibility to host RNA silencing machinery and/or differential stability rates for these vdsRNAs. It is interesting to note that one of these hypo-accumulating regions, the terminal left $(T L)$, contains the terminal conserved hairpin $(\mathrm{TCH})$, a highly conserved region in the HSVd sequence (89) and that besides Hostuviroid is also present in Cocaviroid and Coleviroid genera. In Pospiviroid genus, this region harbors the DNA-dependent RNA polymerase II binding region and the transcription initiation site (90).

Increasing evidence supports the involvement of vd-sRNAs in the down-regulation of host genes via RNA silencing mechanism (23-25, 28, 91, 92). Although we identified diverse HSVd-derived sRNAs predicted as complementary to cucumber transcripts, only two fulfilled the conditions required to be reliably considered as negative regulator of the host gene expression. Our data 
support that vd-sRNAs (180+) and (234-) might be incorporated into the host RNA silencing mediated pathway promoting the RISC-mediated degradation of cucumber homologous to RTM2 and AQP, respectively.

RTM1 and RTM2 genes encode a jacalin-repeat protein and a transmembrane-containing small heat-shock-like (hsp20-like) protein, respectively, and are involved in a functional mechanism responsible of the specific restriction of the long-distance movement of Tobacco etch potyvirus (TEV) in A. thaliana (93). Although the basis of this phenomenon is unclear, it has been proposed that the restriction could be the result of physical blockage of virus entry into, passage through, or exit from the phloem (94). On the other hand, aquaporins are membrane proteins ubiquitously present in all kingdoms of life. Although were originally discovered as water channels, increasing evidences support their involvement in response to biotic stress (95), probably by regulating the transport of H2O2, a reactive oxygen species essential for pathogens defence, in infected plants (96). The precise modes of aquaporin regulation during infection are still unknown (95). However the observation that aquaporin-derived RNAs are differentially recovered from a degradome dataset obtained from tomato plants infected con CEVd (97), suggests that this differential accumulation of aquaporin transcripts might be modulated by alteration in RNA silencing pathways associated to viroid infection. According to the functional characteristics previously proposed for both RTM2 and AQPS, it is feasible to speculate that the vd-sRNA-mediated silencing of their homologous in cucumber might favour the vascular viroid movement (98) and be closely related to interferences in the signalling processes associated to plant defence mechanism. However, further studies are needed in order to validate the reliably of this very exciting idea.

Having established the global landscape of pathogen-derived sRNAs, next we analyzed the influence of the HSVd infection onto the endogenous sRNA population. A first view of the sequence data evidenced that the general profile of cucumber sRNAs is not affected in a significant manner in any of the analyzed time points. Considering sequence accumulation specifically, we observed that the biogenesis of endogenous sRNAs remains relatively stable in response to viroid accumulation, with only 1482 unique sRNAs with significant altered expression at $24 \mathrm{dpi}$. The predominant trend of this response was the up-regulation of the hostderived sRNAs. Differentially recovered sRNAs were not identified at $10 \mathrm{dpi}$, while a considerable proportion of the sRNAs with significant differential expression observed at 17 and $24 \mathrm{dpi}$ were derived from ribosomal transcripts and associated to hypomethylation of rDNA induced by HSVd-infection (discussed in depth below). A similar scenario (characterized by a lower reactivity level) was observed when alteration in miRNA population was analyzed. Seven 
miRNA families were significantly down-regulated in infected plants, and except for miR319 (responsive also at $17 \mathrm{dpi}$ ) the remaining miRNAs were differentially expressed only at $24 \mathrm{dpi}$. The association between miR319 downregulation and infection was also evident considering the number of family members (12 at 24 dpi) that showed comparable differential expression. However, the changes observed in miR319 level were not associated to antagonist alterations in the accumulation of their regulatory targets. It is well established that TEOSINTE BRANCHED1/CYCLOIDEA/PROLIFERATIN CELL FACTOR (TCP) mRNA is a highly conserved target of miR319 in diverse plant species (99) and accumulating evidence has revealed that miR319regulated TCP (MRTCP) genes participate extensively in plant development by regulating hormone metabolism mediated signaling (100). Interestingly, it has been previously reported that tomato plants infected with PSTVd (another member in the Pospiviroidae family) exhibit a complex array of changes affecting hormone signaling (30). However the lack of altered transcriptional activity in infected plants of MRTCP genes, argues against the possibility that such physiological effects can be associated to HSVd infection in cucumber. No significant correlation was observed when the targets of the other six differentially expressed miRNAs where analyzed evidencing that, in coincidence with the described in other plant-viroid models (27, 31, 101-103), the global effects of HSVd infection in cucumber miRNA metabolisms is weak, and generally not related to an evident regulation of their targets.

Since the first viroid was described, it has been proposed that this type of naked exogenous RNAs may act as an abnormal regulator affecting host transcriptional activity (104). Furthermore, the alteration of the accumulation of plant transcripts, at specific infection phases, has been previously reported in diverse viroid-host interactions (30, 38, 40, 103, 105). Our temporal analysis of the evolution of the cucumber transcriptional landscape in response to HSVd infection indicates that although significant transcriptional alterations were observed in the three analyzed time points, the intensity of this response (considering both, the number of differential transcripts and the alterations in the accumulation levels) paralleled the development of the infection, being slight at $10 \mathrm{dpi}$ and more evident at $24 \mathrm{dpi}$. Another particularity associated to the infection time was the trend of the transcriptional response, while the upregulation of host transcripts was the predominant response at $10 \mathrm{dpi}$, the downregulated transcripts were the most recovered from our dataset at 17 and 24 dpi. When the functionality of the differential transcripts was analyzed, it was evident that HSVd-infection is associated to an extensive reprogramming of the host transcriptional activity, affecting cucumber genes involved in diverse cellular functions. This observation is in agreement with previous data obtained from plants infected with two (severe and mild) HSVd-variants, showing 
a complex array of changes in the host transcriptome associated to viroid infection (42). However, a more defined functional response to HSVd-infection was evident when we considered only transcripts differentially expressed at 17 and 24 dpi. Cucumber genes included in this common response were predominantly downregulated and mainly involved in membrane metabolism, transport and response to biotic stress.

Besides transcript accumulation, it is well established that alternative splicing is a regulated process that increases the transcriptome diversity, providing an alternative way to favor the plant adaptation to environmental changes $(106,107)$. However, our knowledge about the alternative processing of host transcripts in response to viroid infection is very limited and only data related to PSTVd-infected tomato plants at late infection has been reported (103). Our results evidenced that the differential exon usage was the predominant transcriptional response during the initial phase of the HSVd-infection (10 dpi). In contrast to the observed for transcripts expression, the global trend (up or down regulation) in exons accumulation was comparable at the three analyzed time points. Only a small proportion $(5,76 \%)$ of the transcripts having AS, also exhibited significant differential accumulation, suggesting that the control of the transcriptional activity and the alternative processing of pre-mRNAs might be complementary mechanisms activated in cucumber plants in response to HSVd infection. Regarding to the functionality of the transcripts showing significant AS in response to HSVd-infection, we observed that although the initial response included cucumber genes involved in many cellular processes, transcripts showing a more constant differential exons usage frequency during infection were predominantly related to primary metabolism. The observation that the AS of transcripts with similar biological function was also reported in PSTVd-infected tomato plants (103), which suggest that the regulation of the plant metabolism mediated by differential exons usage might be a host response mechanism also extended to other nuclear-replicating viroids.

The differential methylation of cytosines is another host regulatory layer susceptible to be affected in response to viroid infection (108). Although, this epigenetic mechanism was first described studying viroid infection in PSTVd-expressing transgenic tobacco plants (109), the global effects of viroid-infection into host DNA methylation, remains yet unexplored (6). The WGBS analysis demonstrated that HSVd infection is associated with significant alterations in the host epigenetic landscape. Under a global viewpoint, the alteration in cytosine methylation was a dynamic phenomenon associated to infection phases (slight global hypomethylation at early infection and significant hypermethylation at $24 \mathrm{dpi}$ ) and sequence context (predominant hypomethylated DMRs at CG context and hypermethylated $\mathrm{CHH}$ ). Studying in detail the regulatory effects of this altered epigenetic landscape; we observed that the changes in 
methylation levels (at CG sequence context) of putative promoter regions and CDS correlate with the transcriptional alterations observed in infected plants. Thus suggesting that these HSVd-associated epigenetic variations may be responsible of the differential expression of certain genes in infected cucumber plants. Previously, it was reported that in cucumber $(49,82)$ and $N$. benthamiana (110) plants, HSVd accumulation is associated to significant hypomethylation of the promoters region in rRNA genes. The results obtained here, besides reinforcing the previous results, demonstrated that the alterations in rDNA methylation are also extended to the totality of the rRNA transcriptional unity.

The integrative analysis of our data supports that in response to HSVd significant alterations are triggered in cucumber plants at different regulatory levels that are closely related to the temporal evolution of the infection process (Fig. 7). Differential exon usage, modulated by alternative splicing, emerges as the predominant response at initial infection stage. It is well established that the alternative processing of the pre-mRNAs increases the diversity of the plant transcriptome and proteome (106). Consequently, this rearrangement of the hosttranscriptome (without new transcriptional activity) might, in similar way to the previously described for other stress conditions (107), allow the immediate fine tuning of the host gene expression in response to HSVd-infection. At subsequent infection phases, the modulation of the plant-response to the viroid will be mainly promoted by alterations in the transcriptional activity that was evidently increased at $17 \mathrm{dpi}$ and being the mostly affected regulatory-layer (considering both, intensity and diversity) at $24 \mathrm{dpi}$. Interestingly, altered transcription shows a temporal coincidence with changes in the methylation profiles of promoter regions in certain cucumber genes. This functional connection permits to speculate about the possibility that the non-immediate transcriptional response to HSVd might be modulated by the host epigenetic changes associated to infection. Finally, it was obvious that the alteration in sRNA and miRNA metabolism played a minor functional role in the recovering of the cell homeostasis in response to HSVd infection.

An interesting emerging question is how HSVd may trigger the regulatory alterations observed in infected plants. It is generally proposed that disease development must be the result of the direct interaction between viroid-RNA and certain host factors (11). For example, it has been recently shown that the direct interaction between two symptomatic viroids (PSTVd and CEVd) and the host translational machinery induce ribosomal stress in tomato plants (111). Assuming this possibility, it may be hypothesized that resembling the described for the arabidopsis long ncRNA ASCO (112), HSVd (an exogenous IncRNA) might bind to specific splicing factors affecting pre-mRNA processing. Regarding transcriptional alterations linked to host epigenetic changes, it 
bioRxiv preprint doi: https://doi.org/10.1101/2022.01.06.475203; this version posted January 6, 2022. The copyright holder for this preprint (which was not certified by peer review) is the author/funder, who has granted bioRxiv a license to display the preprint in perpetuity. It is made available under aCC-BY-ND 4.0 International license.

has been proposed that the direct interaction between viroid-genome and HISTONE DEACETYLASE-6 (HDA6) is responsible of the methylation changes observed in the rDNA of HSVd-infected cucumber plants (48). Consequently, the possibility that the HSVd might modulate the host epigenetic landscape by sequestering key components of the RNA-directed DNA Methylation (RdDM) pathway should not be yet excluded. Alternatively, the specific silencing of certain host genes mediated by vd-sRNAs can also contribute to the global redesign of the host regulatory structure. Further studies focused on a deep analysis of the potential interactions between viroid-RNA and host components will be needed for understanding the ways employed by HSVd to redesign the cucumber regulatory scenario.

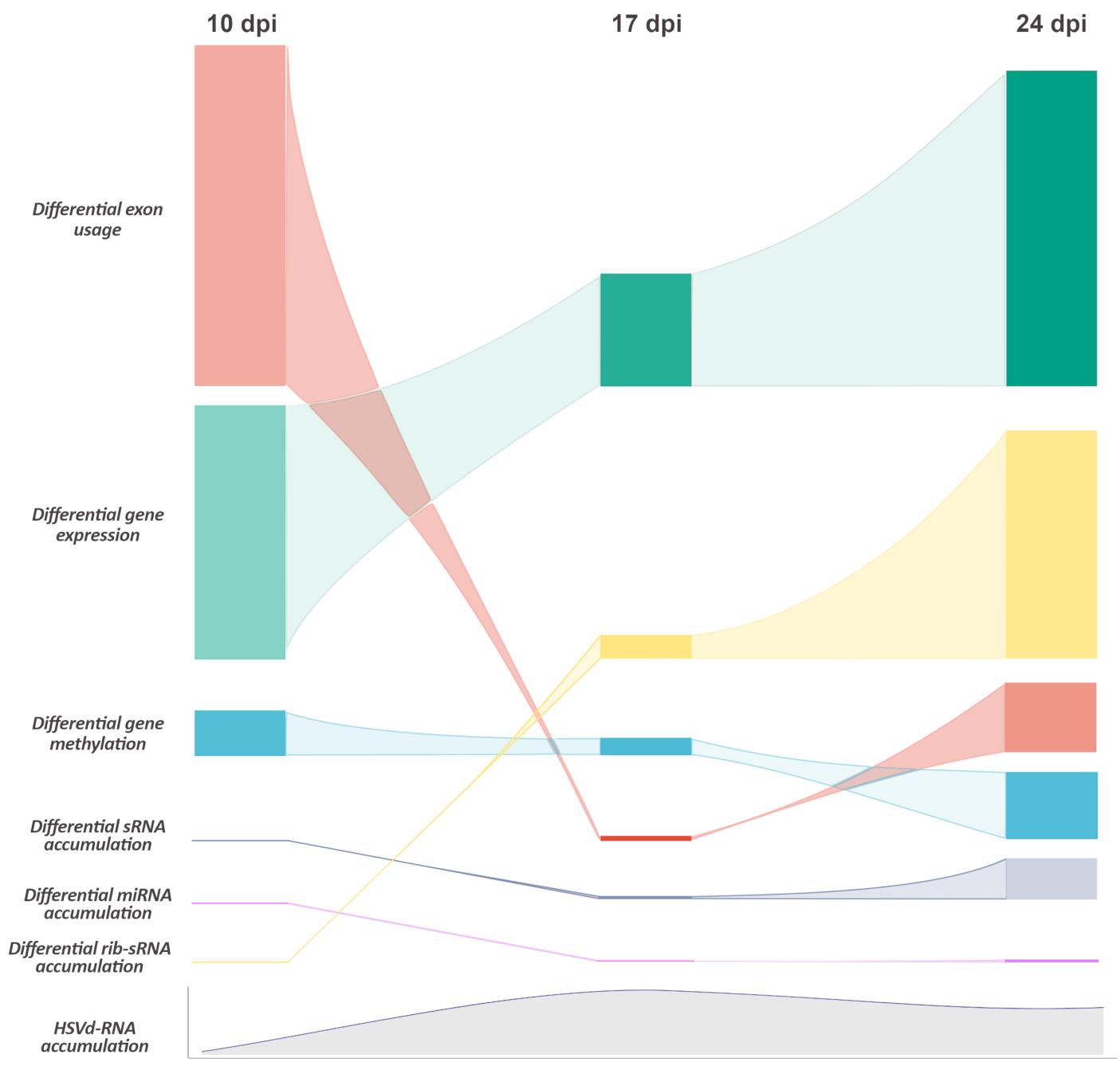

Figure 7: Graphic representation showing the temporal evolution of the global host-regulatory response to HSVd-infection in cucumber plants. The predominant regulatory layers affected in each analyzed infection time are hierarchical represented by the density (box length) estimated by the number of alterations and intensity (box color strength) estimated by the absolute differential value of the response. To represent the density of the alterations in methylation we considered the number of genes with significant DMRs in CG context. In the sRNA group are included all identified classes except rib-sRNAs and miRNAs. The global position in each analyzed timepoint indicates the predominance of the regulatory layer in the global response to infection. Below is also represented the relative accumulation (estimated by transcript recovering) of HSVd in infected plants.

Altogether, the data showed here constitute a comprehensive multiomic approach aimed to provide an overview of the temporal evolution of the host response to HSVd infection. 
Recognizing that this is only the initial step in a long road, we expect that this innovative viewing will contribute to elucidate the mechanistic and the molecular basis of the host changes triggered by viroid infection.

\section{METHODS}

Viroid infection and sample collection

Cucumber plants cv. Marketer at cotyledon stage were inoculated with Hop stunt viroid (Y09352.1) as previously described (54). Both cotyledons were agro-infiltrated with a culture of Agrobacterium tumefaciens strain C58 with 1 of optical density, harbouring pMD201t-HSVd or empty vector, diluted in infiltration buffer (MES $0.1 \mathrm{M}, \mathrm{MgCl} 20.1 \mathrm{M}$ ). Plants were kept in a photoperiod of $16 \mathrm{~h}$ under visible light and $30{ }^{\circ} \mathrm{C}$ (light)/25 ${ }^{\circ} \mathrm{C}$ (darkness). The second leaf after the apex was collected at 10-, 17- and 24-days post inoculation. Each bio-replicate consisted of the leaves from three plants and for each time-point three bio-replicates were sampled of mock and HSVd infected plants.

DNA and RNA extraction and library preparation.

Total RNA was extracted using TRIzol reagent (Life Technologies) following the manufacturer instructions. Genomic DNA was extracted using a CTAB-based extraction method as previously described (55). All libraries were constructed by Novogene Europe (Cambridge, U.K.) according to their standard procedures, including the previous steps of mRNA purification with oligo(dT) beads sRNA purification by size selection.

Small RNA sequencing and analysis

sRNA libraries were sequenced by single end (50 pb). The resulting reads were adapter trimmed and filtered on quality by Cutadapt v2.8(56) and Trimmomatic v0.32 (57), respectively. Additionally, only reads in the 20-25 length range and without indeterminations were kept using a custom script. To study the correlation exhibited by the sRNA expression profiles among the different samples, principal component analysis (PCA) was used. PCA was performed using the prcomp function with scaling in the stats R package v. 4.0.4 (R Core Team 2013). Mann-Whitney-Wilcoxon tests were performed to assess for significant differences in the data clusters for Euclidean distances calculated between groups and among groups with the wilcox.test function in the stats R package. Libraries were aligned to the genomes of HSVd (Y09352.1) and Cucumis sativus Chinese Long V3 (58) to determine the provenance of every 
SRNA. Only sequences that aligned with no mismatches exclusively either to the viroid or the plant genome with a minimum of 0.5 RPMs were considered for further analysis. Additionally, for estimating the size abundance, only samples with all the sRNA lengths were considered. Moreover, HSVd-sRNAs were classified according to their origin (positive or negative viroid strand) considering unique sequences and also the accumulation (rpm). Then, an exact binomial test was performed to assess the significance. Differential expression of cucumber sRNAs was estimated using two R packages DESeq2 (59) and edgeR (60) for pairwise differential expression analysis of expression data. Differentially expressed sRNAs were filtered out using two criteria: (i) adjusted $p$-value $\leq 0.05$ and (ii) RPMs $\geq 5$ for at least two libraries in stressed or control samples. P-values were adjusted by False Discovery Rate (FDR). miRNAs were annotated from previously described miRNAs in cucumber (61) and validated with the precursors deposited in RNA central (62). The rest of sRNA categories were annotated using RNA central and sRNAanno (63).

mRNA sequencing and analysis

RNA sequencing paired reads (150 bp) were aligned to the cucumber genome using STAR (64). For non-annotated transcripts, a de novo assembly was performed using Trinity (65). 15589 transcripts non-overlapping with the gene annotation of Chinese Long V3 (58) were aligned with gmap allowing chimeric alignments (66) and a custom annotation of non-coding transcripts was obtained using bedtools (67). HTSeq-counts (68) was firstly used to count reads per annotated genes using 10 as a minimum alignment quality and secondly of the novel annotated transcripts with a minimum alignment quality of 0 and counting all reads ambiguously mapped in both categories and wherever they overlap (in order to account for potential transposable elements). The count tables were used in DESeq2 (59) to infer significant expression, considering an adjusted p-value under 0.05 for significance. Clustering analysis of the transcripts with significant differential expression in the three time-points was calculated with stats R-package. Firstly, the optimal number of clusters was determined using the complete method in the NbClust R-package considering Euclidean distances (69). Secondly, the function 'kmeans' of stats was applied with one million iterations. The resulting clusters (grouping transcripts with similar evolution of the differential expression profiles over the infection) were generated by ggplot2 (70).

Moreover, to account for differential exon usage, the package DEXSeq was employed (71), also considering an adjusted p-value under 0.05 for significance. Volcano plots were created using ggplot2 (70). GO term enrichment analysis was performed using the tool from Cucurbit 
Genomics Database (CuGenDB) (58). p-values were corrected using false discovery rate (FDR) and the cutoff p-value for significant represented terms was 0.05 .

\section{5' PARE analysis}

Libraries for 5' parallel analysis of RNA end (PARE) were constructed following the protocol previously described with minor modifications (72). Poly $(A+)$ RNA ( $\approx 1000 \mathrm{ng})$ was purified from total RNA from each mock and HSVd replicate of 24 dpi using the GenElute Direct mRNA Miniprep Kit (Sigma-Aldrich). A 5' RNA oligonucleotide adaptor (PARE 5' Adapter) containing a Mme I recognition site was ligated to the 5'-phosphate of the truncated poly $(A+)$ RNA by T4 RNA ligase. The ligated products were purified by ethanol precipitation and subjected to a reverse transcription reaction with an oligo dT primer with a known tail (dT primer). Following RNA degradation by alkaline lysis, the cDNA was amplified by PCR using adaptor and oligo dTspecific primers (I5(1)-F and GR3', respectively), digested with Mme I and ligated to a 3' double DNA adaptor (dsDNA-top and dsDNA-bottom). The ligated products were separated by electrophoresis in polyacrylamide, and those with the expected size were gel-purified and amplified with I5(2)-F and the corresponding indexed I7-R. PCR products of the expected size were gel-purified and subjected to sequencing by Illumina technology. Primers used are listed in Supplemental Table S1.

The resulting reads were adapter trimmed by Cutadapt v2.8 (56) and filtered on quality and length by fastp v0.23.1 (73), including only reads in the 19-21 length range. The sequence quality was checked by FastQC v0.11.5. Then, libraries were aligned on the assembled transcriptome of Cucumis sativus Chinese Long V3 (58). Degradome sequences were filtered according to the following: i) alignment on a differentially downregulated transcript at 24 dpi, ii) presence in at least two HSVd libraries and absence in mock libraries and iii) match with the predicted target site of a HSVd-sRNA according to the psRNATarget prediction (74).

\section{Reporter assay and RT-qPCR}

The predicted sequences to be targeted by HSVd-sRNAs were cloned upstream of the yellow fluorescent protein (YFP) by self-hybridizing oligonucleotides to obtain the reporters REPCsaV3_1G041760 and REP-CsaV3_6G040100 (listed in Supplemental Table S1). These oligonucleotides comprise the start codon ATG followed by eight codons (in frame with the YFP) of the cucumber transcript which include the 21 nucleotides predicted by psRNATarget (74). Nicotiana benthamiana leaves were agro-infiltrated with a mixture of the reporter with either the binary plasmid expressing HSVd (54) or the empty plasmid (mock) at optical 
densities $600 \mathrm{~nm}$ of 0.05 and 0.5 , respectively. Fluorescence was observed at three days postinoculation with Leica MSV269 stereomicroscope with an excitation wavelength of $485 \mathrm{~nm}$ and images were taken with Leica DFC7000T with identical parameters focusing the infiltration circle. RNA was extracted from the infiltrated leaves after fluorescence observation using TRIzol reagent (Invitrogen, Carlsbad, CA, USA). First-strand cDNA was synthesized by reverse transcription using oligo(dT)18 and RevertAid cDNA Synthesis Kit (Thermo Scientific ${ }^{\mathrm{TM}}$, Waltham, MA, USA). qRT-PCR assays were performed using PyroTaq EvaGreen mix Plus (ROX) (CulteK Molecular Bioline) according to the manufacturer's instructions. All analyses were performed in triplicate on an ABI 7500 Fast-Real Time qPCR instrument (Applied Biosystems). Relative YFP expression (YFP-F, YFP-R) was determined by using the comparative $\Delta \triangle \mathrm{CT}$ method and normalized to the geometric mean of F-box (HK-FBOX-F, HK-FBOX-R). The statistical significance of the observed differences was evaluated by the paired t-Test.

\section{Bisulfite sequencing analysis and DMR identification}

Whole Genome Bisulfite Sequencing (WGBS) libraries were sequenced by paired end (150 pb). The resulting reads were adapter trimmed and filtered on quality by Trimgalore v0.6.6 . Additionally, 10 bases from $5^{\prime}$ ends were trimmed from reads using the same software. The sequence quality was checked by FastQC v0.11.9. The mean conversion rate for the 36 libraries was above 99\%. Clean reads were mapped to the reference genome using bismark (75) allowing one mismatch per 25 nucleotides length seed. Alignments at the same position were removed using deduplicate_bismark script, including forward and reverse reads. The bismark_methylation_extractor script was used to extract the methylation call for every single cytosine analyzed and obtain a genome-wide methylation report discriminating by context (CG/CHG/CHH).

The analysis of differentially methylated regions (DMR) was carried out with the R-package DMRcaller (76) dividing the genome in equal bins of $50 \mathrm{pb}$ and pooling the samples of the same condition. The DMR were then computed by performing Fisher's exact test between the number of methylated reads and the total number of reads in both conditions for each bin. The obtained p-values were then adjusted for multiple testing using Benjamini-Hochberg's method to control the false discovery (77). The criteria chosen to consider a bin as a DMR were the following: i) adjusted p-value $<=0.05$, ii) at least three cytosines in the specified context, iii) more than a $15 \%$ methylation difference between the two conditions and iiii) at least an average number of reads of eight. Finally bins that were at less than $300 \mathrm{pb}$ were joined. 
In addition to the DMRs identification, overall methylation was calculated as the mean of the percentage methylation of cytosines distinguishing among contexts. Those cytosines covered by less than eight reads were discarded. Next, a heat-map representing the genome-wide methylation difference between the two conditions was performed. First, cytosine methylation percentages were grouped in 3000 nucleotide size windows. Second, the methylation difference was calculated according to time and context. Third, the methylation difference of windows meeting the following terms was set to zero: i) mock or HSVd show zero reads coverage and ii) mock and HSVd show less than eight reads coverage. Methylation difference values less than $-15 \%$ or greater than $15 \%$ were considered as $-15 \%$ and $15 \%$, respectively. Red and blue colors represented the hypomethylation and hypermethylation in HSVd. Both approaches were also carried out for the ribosomal sequence.

\section{FUNDING}

This work was supported by the Spanish Ministry of Economy and Competitiveness (cosupported by FEDER) Grants PID2019-104126RB-I00 (GG) and PID2020-115571RB-I00 (VP). J.M.M. was recipient of a pre-doctoral contract ACIF-2017-114 from the Generalitat Valenciana. The funders had no role in the experiment design, data analysis, decision to publish, or preparation of the manuscript.

\section{AUTHOR CONTRIBUTIONS}

J.M.M. performed the experiments. P.V.B, J.C.S. and J.M.M performed the bioinformatic analysis. J.M.M., V.P and G.G.: designed the experiment. J.M.M., P.V.B, J.C.S., V.P. and G.G. analyzed and discussed the results. G.G. Conceived the general experiment and drafted the manuscript. All authors read, revise and approved the final manuscript.

\section{COMPETING INTERESTS}

The author(s) declare no competing interests.

\section{REFERENCES}

1. Di Serio,F., Li,S.-F., Pallás,V., Owens,R.A., Randles,J.W., Sano,T., Verhoeven,J.T.J., Vidalakis,G. and Flores,R. (2017) Chapter 13 - Viroid Taxonomy. In Hadidi,A., Flores,R., Randles,J.W., Palukaitis,P.B.T.-V. and S. (eds). Academic Press, Boston, pp. 135-146.

2. Ding,B. (2009) The Biology of Viroid-Host Interactions. Annu. Rev. Phytopathol., 47, 105-131.

3. Adkar-Purushothama,C.R. and Perreault,J.-P. (2020) Current overview on viroid-host interactions. WIRES RNA, 11, e1570.

4. Gómez,G. and Pallás,V. (2013) Viroids: A light in the darkness of the IncRNA-directed regulatory networks in plants. New Phytol., 198, 10-15. 
5. Gago-Zachert,S. (2016) Viroids, infectious long non-coding RNAs with autonomous replication. Virus Res., 212, 12-24.

6. Navarro,B., Flores,R. and Di Serio,F. (2021) Advances in Viroid-Host Interactions. Annu. Rev. Virol., 8, 305-325.

7. Yaish,M.W. (2017) Editorial: Epigenetic Modifications Associated with Abiotic and Biotic Stresses in Plants: An Implication for Understanding Plant Evolution . Front. Plant Sci. , 8, 1983.

8. Baulcombe,D.C. and Dean,C. (2014) Epigenetic regulation in plant responses to the environment. Cold Spring Harb. Perspect. Biol., 6, a019471-a019471.

9. Annacondia,M.L., Magerøy,M.H. and Martinez,G. (2018) Stress response regulation by epigenetic mechanisms: changing of the guards. Physiol. Plant., 162, 239-250.

10. Wilkins,O., Hafemeister,C., Plessis,A., Holloway-Phillips,M.-M., Pham,G.M., Nicotra,A.B., Gregorio,G.B., Jagadish,S.V.K., Septiningsih,E.M., Bonneau,R., et al. (2016) EGRINs (Environmental Gene Regulatory Influence Networks) in Rice That Function in the Response to Water Deficit, High Temperature, and Agricultural Environments. Plant Cell, 28, 2365-2384.

11. Adkar-Purushothama,C.R. and Perreault,J.-P. (2019) Current overview on viroid-host interactions. Wiley Interdiscip. Rev. RNA, 10.1002/wrna.1570.

12. Itaya,A., Zhong,X., Bundschuh,R., Qi,Y., Wang,Y., Takeda,R., Harris,A.R., Molina,C., Nelson,R.S. and Ding,B. (2007) A Structured Viroid RNA Serves as a Substrate for Dicer-Like Cleavage To Produce Biologically Active Small RNAs but Is Resistant to RNA-Induced Silencing Complex-Mediated Degradation. J. Virol., 81, 2980-2994.

13. Gómez,G. and Pallás,V. (2007) Mature monomeric forms of Hop stunt viroid resist RNA silencing in transgenic plants. Plant J., 51.

14. Elena,S.F., Gómez,G. and Daròs,J.A. (2009) Evolutionary constraints to viroid evolution. Viruses, 1, 241-254.

15. Martinez de Alba,A.E., Flores,R. and Hernandez,C. (2002) Two Chloroplastic Viroids Induce the Accumulation of Small RNAs Associated with Posttranscriptional Gene Silencing. J. Virol., 76, 13094-13096.

16. Itaya,A., Folimonov,A., Matsuda,Y., Nelson,R.S. and Ding,B. (2001) Potato spindle tuber viroid as inducer of RNA silencing in infected tomato. Mol. Plant-Microbe Interact., 14, 1332-1334.

17. Papaefthimiou,I., Hamilton,A., Denti,M., Baulcombe,D., Tsagris,M. and Tabler,M. (2001) Replicating potato spindle tuber viroid RNA is accompanied by short RNA fragments that are characteristic of post-transcriptional gene silencing. Nucleic Acids Res., 29, 2395-400.

18. Wang,M.B., Bian,X.Y., Wu,L.M., Liu,L.X., Smith,N.A., Isenegger,D., Wu,R.M., Masuta,C., Vance,V.B., Watson,J.M., et al. (2004) On the role of RNA silencing in the pathogenicity and evolution of viroids and viral satellites. Proc. Natl. Acad. Sci. U. S. A., 101, 3275-3280. 
19. Gómez,G., Martínez,G. and Pallás,V. (2009) Interplay between viroid-induced pathogenesis and RNA silencing pathways. Trends Plant Sci., 14, 264-9.

20. Adkar-Purushothama,C.R. and Perreault,J.P. (2018) Alterations of the viroid regions that interact with the host defense genes attenuate viroid infection in host plant. RNA Biol., 15.

21. Adkar-Purushothama,C.R., Iyer,P.S. and Perreault,J.-P. (2017) Potato spindle tuber viroid infection triggers degradation of chloride channel protein CLC-b-like and Ribosomal protein S3a-like mRNAs in tomato plants. Sci. Rep., 7, 8341.

22. Adkar-Purushothama,C.R., Sano,T. and Perreault,J.-P. (2018) Viroid-derived small RNA induces early flowering in tomato plants by RNA silencing. Mol. Plant Pathol., $19,2446-2458$.

23. Adkar-Purushothama,C.R., Brosseau,C., Giguère,T., Sano,T., Moffett,P. and Perreault,J.-P. (2015) Small RNA Derived from the Virulence Modulating Region of the Potato spindle tuber viroid Silences callose synthase Genes of Tomato Plants. Plant Cell, 27, 2178-2194.

24. Avina-Padilla,K., Martinez de la Vega,O., Rivera-Bustamante,R., MartinezSoriano,J.P., Owens,R.A., Hammond,R.W. and Vielle-Calzada,J.-P. (2015) In silico prediction and validation of potential gene targets for pospiviroid-derived small RNAs during tomato infection. Gene, 564, 197-205.

25. Wang,Y., Shibuya,M., Taneda,A., Kurauchi,T., Senda,M., Owens,R.A. and Sano,T. (2011) Accumulation of Potato spindle tuber viroid-specific small RNAs is accompanied by specific changes in gene expression in two tomato cultivars. Virology, 413, 72-83.

26. Bao,S., Owens,R.A., Sun,Q., Song,H., Liu,Y., Eamens,A.L., Feng,H., Tian,H., Wang,M.B. and Zhang,R. (2019) Silencing of transcription factor encoding gene StTCP23 by small RNAs derived from the virulence modulating region of potato spindle tuber viroid is associated with symptom development in potato. PLOS Pathog., 15, e1008110.

27. Navarro,B., Gisel,A., Serra,P., Chiumenti,M. and Di Serio,F. (2021) Degradome Analysis of Tomato and Nicotiana benthamiana Plants Infected with Potato Spindle Tuber Viroid. Int. J. Mol. Sci., 22.

28. Navarro,B., Gisel,A., Rodio,M.E., Delgado,S., Flores,R. and Di Serio,F. (2012) Small RNAs containing the pathogenic determinant of a chloroplast-replicating viroid guide the degradation of a host mRNA as predicted by RNA silencing. Plant J., 70, 991-1003.

29. Diermann,N., Matoušek,J., Junge,M., Riesner,D. and Steger,G. (2010) Characterization of plant miRNAs and small RNAs derived from potato spindle tuber viroid (PSTVd) in infected tomato. 391, 1379-1390.

30. Owens,R.A., Tech,K.B., Shao,J.Y., Sano,T. and Baker,C.J. (2012) Global analysis of tomato gene expression during potato spindle tuber viroid infection reveals a complex array of changes affecting hormone signaling. Mol. Plant-Microbe 
Interact., 25, 582-598.

31. Tsushima,D., Adkar-Purushothama,C.R., Taneda,A. and Sano,T. (2015) Changes in relative expression levels of viroid-specific small RNAs and microRNAs in tomato plants infected with severe and mild symptom-inducing isolates of Potato spindle tuber viroid. J. Gen. Plant Pathol., 81, 49-62.

32. Sanz-Carbonell,A., Marques,M.C., Bustamante,A., Fares,M.A., Rodrigo,G. and Gomez,G. (2019) Inferring the regulatory network of the miRNA-mediated response to biotic and abiotic stress in melon. BMC Plant Biol., 19, 78.

33. Dynamic architecture and regulatory implications of the miRNA network underlying the response to stress in melon.

34. Lavagi-Craddock,I., Bodaghi,S. and Vidalakis,G. (2021) Next-Generation Sequencing Identification and Characterization of MicroRNAs in Dwarfed Citrus Trees Infected With Citrus Dwarfing Viroid in High-Density Plantings. Front. Microbiol., 12.

35. Hadjieva,N., Ivanova,D.P., Yahubyan,G. and Baev,V. (2021) MicroRNA expression dynamics reshape the cultivar-specific response of pepper (Capsicum annuum L.) to potato spindle tuber viroid (PSTVd) infection. Sci. Hortic., 278.

36. Wang,X., Li,N., Li,W., Gao,X., Cha,M., Qin,L. and Liu,L. (2020) Advances in Transcriptomics in the Response to Stress in Plants. Glob. Med. Genet., 7, 30-34.

37. Waters,A.J., Makarevitch,I., Noshay,J., Burghardt,L.T., Hirsch,C.N., Hirsch,C.D. and Springer,N.M. (2017) Natural variation for gene expression responses to abiotic stress in maize. Plant J., 89, 706-717.

38. RIZZA,S., CONESA,A.N.A., JUAREZ,J., CATARA,A., NAVARRO,L., DURAN-VILA,N. and ANCILLO,G. (2012) Microarray analysis of Etrog citron (Citrus medica L.) reveals changes in chloroplast, cell wall, peroxidase and symporter activities in response to viroid infection. Mol. Plant Pathol., 13, 852-864.

39. Wiesyk,A., Fogtman,A., Lirski,M. and Zagorski-Ostoja,W. (2019) Root Transcriptomic Analysis Reveals Global Changes Induced by Systemic Infection of Solanum lycopersicum with Mild and Severe Variants of Potato Spindle Tuber Viroid. Viruses., 11.

40. Herranz,M.C., Niehl,A., Rosales,M., Fiore,N., Zamorano,A., Granell,A. and Pallas,V. (2013) A remarkable synergistic effect at the transcriptomic level in peach fruits doubly infected by prunus necrotic ringspot virus and peach latent mosaic viroid. Virol. J., 10, 164.

41. Kappagantu,M., Bullock,J.M., Nelson,M.E. and Eastwell,K.C. (2017) Hop stunt viroid: Effect on host (Humulus lupulus) transcriptome and its interactions with hop powdery mildew (Podospheara macularis). Mol. Plant-Microbe Interact., 30, 842851.

42. Xia,C., Li,S., Hou,W., Fan,Z., Xiao,H., Lu,M., Sano,T. and Zhang,Z. (2017) Global transcriptomic changes induced by infection of cucumber (Cucumis sativus L.) with mild and severe variants of hop stunt viroid. Front. Microbiol., 8. 
43. Štajner,N., Radišek,S., Mishra,A.K., Nath,V.S., Matoušek,J. and Jakše,J. (2019) Evaluation of Disease Severity and Global Transcriptome Response Induced by Citrus bark cracking viroid, Hop latent viroid, and Their Co-Infection in Hop (Humulus lupulus L.). Int. J. Mol. Sci., 20, 3154.

44. Katsarou,K., Wu,Y., Zhang,R., Bonar,N., Morris,J., Hedley,P.E., Bryan,G.J., Kalantidis,K. and Hornyik,C. (2016) Insight on Genes Affecting Tuber Development in Potato upon Potato spindle tuber viroid (PSTVd) Infection. PLoS One, 11, e0150711-e0150711.

45. Wang,C., Wang,C., Zou,J., Yang,Y., Li,Z. and Zhu,S. (2019) Epigenetics in the plantvirus interaction. Plant Cell Rep., 38, 1031-1038.

46. Gómez-Díaz,E., Jordà,M., Peinado,M.A. and Rivero,A. (2012) Epigenetics of HostPathogen Interactions: The Road Ahead and the Road Behind. PLoS Pathog., 8, e1003007.

47. Martinez,G., Castellano,M., Tortosa,M., Pallas,V. and Gomez,G. (2014) A pathogenic non-coding RNA induces changes in dynamic DNA methylation of ribosomal RNA genes in host plants. Nucleic Acids Res., 42, 1553-62.

48. Castellano,M., Pallas,V. and Gomez,G. (2016) A pathogenic long noncoding RNA redesigns the epigenetic landscape of the infected cells by subverting host Histone Deacetylase 6 activity. New Phytol., 211, 1311-1322.

49. Castellano,M., Martinez,G., Marques,M.C., Moreno-Romero,J., Köhler,C., Pallas,V. and Gomez,G. (2016) Changes in the DNA methylation pattern of the host male gametophyte of viroid-infected cucumber plants. J. Exp. Bot., 67, 5857-5868.

50. Corrêa,R.L., Sanz-Carbonell,A., Kogej,Z., Müller,S.Y., Ambrós,S., López-Gomollón,S., Gómez,G., Baulcombe,D.C. and Elena,S.F. (2020) Viral Fitness Determines the Magnitude of Transcriptomic and Epigenomic Reprogramming of Defense Responses in Plants. Mol. Biol. Evol., 10.1093/molbev/msaa091.

51. Deleris,A., Halter,T. and Navarro,L. (2016) DNA Methylation and Demethylation in Plant Immunity. Annu. Rev. Phytopathol., 54, 579-603.

52. Zhu,Q.-H., Shan,W.-X., Ayliffe,M.A. and Wang,M.-B. (2016) Epigenetic Mechanisms: An Emerging Player in Plant-Microbe Interactions. Mol. Plant-Microbe Interact., 29, 187-196.

53. Marquez-Molins,J., Gomez,G. and Pallas,V. (2021) Hop stunt viroid: A polyphagous pathogenic RNA that has shed light on viroid-host interactions. Mol. Plant Pathol., $22,153-162$.

54. Marquez-Molins,J., Navarro,J.A., Pallas,V. and Gomez,G. (2019) Highly efficient construction of infectious viroid-derived clones. Plant Methods, 15, 87.

55. Healey,A., Furtado,A., Cooper,T. and Henry,R.J. (2014) Protocol: A simple method for extracting next-generation sequencing quality genomic DNA from recalcitrant plant species. Plant Methods, 10, 1-8.

56. Martin,M. (2011) Cutadapt removes adapter sequences from high-throughput 
sequencing reads. EMBnet.journal; Vol 17, No 1 Next Gener. Seq. Data Anal. 10.14806/ej.17.1.200.

57. Bolger,A.M., Lohse,M. and Usadel,B. (2014) Trimmomatic: a flexible trimmer for Illumina sequence data. Bioinformatics, 30, 2114-2120.

58. Zheng,Y., Wu,S., Bai,Y., Sun,H., Jiao,C., Guo,S., Zhao,K., Blanca,J., Zhang,Z., Huang,S., et al. (2019) Cucurbit Genomics Database (CuGenDB): a central portal for comparative and functional genomics of cucurbit crops. Nucleic Acids Res., 47, D1128-D1136.

59. Love,M.I., Huber,W. and Anders,S. (2014) Moderated estimation of fold change and dispersion for RNA-seq data with DESeq2. Genome Biol., 15, 550.

60. Robinson,M.D., McCarthy,D.J. and Smyth,G.K. (2010) edgeR: a Bioconductor package for differential expression analysis of digital gene expression data. Bioinformatics, 26, 139.

61. Xu,X., Zhong,C., Tan,M., Song,Y., Qi,X., Xu,Q. and Chen,X. (2020) Identification of MicroRNAs and Their Targets That Respond to Powdery Mildew Infection in Cucumber by Small RNA and Degradome Sequencing. Front. Genet., 11, 246.

62. Sweeney,B.A., Petrov,A.I., Burkov,B., Finn,R.D., Bateman,A., Szymanski,M., Karlowski,W.M., Gorodkin,J., Seemann,S.E., Cannone,J.J., et al. (2019) RNAcentral: A hub of information for non-coding RNA sequences. Nucleic Acids Res., 47, D221D229.

63. Chen,C., Li,J., Feng,J., Liu,B., Feng,L., Yu,X., Li,G., Zhai,J., Meyers,B.C. and Xia,R. (2021) sRNAanno-a database repository of uniformly annotated small RNAs in plants. Hortic. Res. $202181,8,1-8$.

64. Dobin,A., Davis,C.A., Schlesinger,F., Drenkow,J., Zaleski,C., Jha,S., Batut,P., Chaisson,M. and Gingeras,T.R. (2013) STAR: ultrafast universal RNA-seq aligner. Bioinformatics, 29, 15-21.

65. Grabherr,M.G., Haas,B.J., Yassour,M., Levin,J.Z., Thompson,D.A., Amit,I., Adiconis,X., Fan,L., Raychowdhury,R., Zeng,Q., et al. (2011) Trinity: reconstructing a full-length transcriptome without a genome from RNA-Seq data. Nat. Biotechnol., 29, 644.

66. Wu,T.D. and Watanabe,C.K. (2005) GMAP: a genomic mapping and alignment program for mRNA and EST sequences. Bioinformatics, 21, 1859-1875.

67. Quinlan,A.R. and Hall,I.M. (2010) BEDTools: a flexible suite of utilities for comparing genomic features. Bioinformatics, 26, 841-842.

68. Anders,S., Pyl,P.T. and Huber,W. (2015) HTSeq-A Python framework to work with high-throughput sequencing data. Bioinformatics, 31, 166-169.

69. Charrad,M., Ghazzali,N., Boiteau,V. and Niknafs,A. (2014) Nbclust: An R package for determining the relevant number of clusters in a data set. J. Stat. Softw., 61, 1-36.

70. Wickham,H. (2011) ggplot2. Wiley Interdiscip. Rev. Comput. Stat., 3, 180-185.

71. Anders,S., Reyes,A. and Huber,W. (2012) Detecting differential usage of exons from 
RNA-seq data. Genome Res., 22, 2008-2017.

72. Zhai,J., Arikit,S., Simon,S.A., Kingham,B.F. and Meyers,B.C. (2014) Rapid construction of parallel analysis of RNA end (PARE) libraries for Illumina sequencing. Methods, $67,84-90$.

73. Chen,S., Zhou,Y., Chen,Y. and Gu,J. (2018) fastp: an ultra-fast all-in-one FASTQ preprocessor. Bioinformatics, 34, i884-i890.

74. Dai,X., Zhuang,Z. and Zhao,P.X. (2018) psRNATarget: a plant small RNA target analysis server (2017 release). Nucleic Acids Res., 46, W49-W54.

75. Krueger,F. and Andrews,S.R. (2011) Bismark: a flexible aligner and methylation caller for Bisulfite-Seq applications. Bioinformatics, 27, 1571-1572.

76. Catoni,M., Tsang,J.M., Greco,A.P. and Zabet,N.R. (2018) DMRcaller: a versatile R/Bioconductor package for detection and visualization of differentially methylated regions in CpG and non-CpG contexts. Nucleic Acids Res., 10.1093/nar/gky602.

77. Benjamini,Y. and Hochberg,Y. (1995) Controlling the False Discovery Rate: A Practical and Powerful Approach to Multiple Testing. J. R. Stat. Soc. Ser. B, 57, 289-300.

78. Ling,J., Luo,Z., Liu,F., Mao,Z., Yang,Y. and Xie,B. (2017) Genome-wide analysis of microRNA targeting impacted by SNPs in cucumber genome. BMC Genomics, 18, 275.

79. Xu,X., Wang,K., Pan,J. and Chen,X. (2019) Small RNA sequencing identifies cucumber miRNA roles in waterlogging-triggered adventitious root primordia formation. Mol. Biol. Rep., 46, 6381-6389.

80. Zhang,Z., Xia,C., Matsuda,T., Taneda,A., Murosaki,F., Hou,W., Owens,R.A., Li,S. and Sano,T. (2020) Effects of Host-Adaptive Mutations on Hop Stunt Viroid Pathogenicity and Small RNA Biogenesis. Int. J. Mol. Sci., 21, 7383.

81. Mandadi,K.K. and Scholthof,K.-B.G. (2015) Genome-wide analysis of alternative splicing landscapes modulated during plant-virus interactions in Brachypodium distachyon. Plant Cell, 27, 71-85.

82. Martinez,G., Castellano,M., Tortosa,M., Pallas,V. and Gomez,G. (2014) A pathogenic non-coding RNA induces changes in dynamic DNA methylation of ribosomal RNA genes in host plants. Nucleic Acids Res., 42, 1553-1562.

83. Henras,A.K., Soudet,J., Gérus,M., Lebaron,S., Caizergues-Ferrer,M., Mougin,A. and Henry,Y. (2008) The post-transcriptional steps of eukaryotic ribosome biogenesis. Cell. Mol. Life Sci., 65, 2334-2359.

84. Earley,K.W., Pontvianne,F., Wierzbicki,A.T., Blevins,T., Tucker,S., Costa-Nunes,P., Pontes,O. and Pikaard,C.S. (2010) Mechanisms of HDA6-mediated rRNA gene silencing: Suppression of intergenic Pol II transcription and differential effects on maintenance versus siRNA-directed cytosine methylation. Genes Dev., 24, 11191132.

85. Navarro,B., Pantaleo,V., Gisel,A., Moxon,S., Dalmary,T., Bisztray,G., Di Serio,F. and 
Burgyán,J. (2009) Deep sequencing of viroid-derived small RNAs from grapevine provides new insights on the role of RNA silencing in plant-viroid interaction. PLOS One, 4 .

86. Martinez,G., Donaire,L., Llave,C., Pallas,V. and Gomez,G. (2010) High-throughput sequencing of Hop stunt viroid-derived small RNAs from cucumber leaves and phloem. Mol. Plant Pathol., 11.

87. Sano,T. and Matsuura,Y. (2004) Accumulation of short interfering RNAs characteristic of RNA silencing precedes recovery of tomato plants from severe symptoms of Potato spindle tuber viroid infection. J. Gen. Plant Pathol., 70, 50-53.

88. Di Serio,F., Gisel,A., Navarro,B., Delgado,S., Martínez de Alba,A.-E., Donvito,G. and Flores, R. (2009) Deep sequencing of the small RNAs derived from two symptomatic variants of a chloroplastic viroid: implications for their genesis and for pathogenesis. PLoS One, 4, e7539-e7539.

89. Amari,K., Gomez,G., Myrta,A., Di Terlizzi,B. and Pallás,V. (2001) The molecular characterization of 16 new sequence variants of Hop stunt viroid reveals the existence of invariable regions and a conserved hammerhead-like structure on the viroid molecule. J. Gen. Virol., 82, 953-962.

90. Sano,T. (2021) Progress in 50 years of viroid research-Molecular structure, pathogenicity, and host adaptation. Proc. Jpn. Acad. Ser. B. Phys. Biol. Sci., 97, 371-401.

91. Eamens,A.L., Smith,N.A., Dennis,E.S., Wassenegger,M. and Wang,M.-B. (2014) In Nicotiana species, an artificial microRNA corresponding to the virulence modulating region of Potato spindle tuber viroid directs RNA silencing of a soluble inorganic pyrophosphatase gene and the development of abnormal phenotypes. Virology, 450-451, 266-277.

92. Innate immunity activation and RNAi interplay in citrus exocortis viroid-Tomato pathosystem (2018) Viruses., 10, 587.

93. Kloth,K.J. and Kormelink,R. (2020) Defenses against Virus and Vector: A PhloemBiological Perspective on RTM- and SLI1-Mediated Resistance to Potyviruses and Aphids. Viruses, 12, 129.

94. Whitham,S.A., Anderberg,R.J., Chisholm,S.T. and Carrington,J.C. (2000) Arabidopsis RTM2 gene is necessary for specific restriction of tobacco etch virus and encodes an unusual small heat shock-like protein. Plant Cell, 12, 569-582.

95. Afzal,Z., Howton,T., Sun,Y. and Mukhtar,M. (2016) The Roles of Aquaporins in Plant Stress Responses. J. Dev. Biol., 4, 9.

96. Dynowski,M., Schaaf,G., Loque,D., Moran,O. and Ludewig,U. (2008) Plant plasma membrane water channels conduct the signalling molecule $\mathrm{H} 2 \mathrm{O} 2$. Biochem. J., $414,53-61$.

97. Thibaut,O. and Claude,B. (2018) Innate Immunity Activation and RNAi Interplay in Citrus Exocortis Viroid-Tomato Pathosystem. Viruses, 10, 587. 
98. Pallás,V. and Gómez,G. (2017) Chapter 8 - Viroid Movement. In Hadidi,A., Flores,R., Randles,J.W., Palukaitis,P.B.T.-V. and S. (eds). Academic Press, Boston, pp. 83-91.

99. Palatnik,J.F., Wollmann,H., Schommer,C., Schwab,R., Boisbouvier,J., Rodriguez,R., Warthmann,N., Allen,E., Dezulian,T., Huson,D., et al. (2007) Sequence and Expression Differences Underlie Functional Specialization of Arabidopsis MicroRNAs miR159 and miR319. Dev. Cell, 13, 115-125.

100. Fang,Y., Zheng,Y., Lu,W., Li,J., Duan,Y., Zhang,S. and Wang,Y. (2021) Roles of miR319-regulated TCPs in plant development and response to abiotic stress. Crop J., 9, 17-28.

101. Martín,R., Arenas,C., Daròs,J.-A., Covarrubias,A., Reyes,J.L. and Chua,N.-H. (2007) Characterization of small RNAs derived from Citrus exocortis viroid (CEVd) in infected tomato plants. Virology, 367, 135-146.

102. Alazem,M., Al-Tuwariqi,H.Z., Barba,M., Boubourakas,I.N., Çağlayan,K., Candresse,T., Conejero,V., Dadami,E., Dalakouras,A., Daròs,J.-A., et al. (2017) List of Contributors. In Hadidi,A., Flores,R., Randles,J.W., Palukaitis,P.B.T.-V. and S. (eds). Academic Press, Boston, pp. xxv-xxviii.

103. Zheng,Y., Wang,Y., Ding,B. and Fei,Z. (2017) Comprehensive Transcriptome Analyses Reveal that Potato Spindle Tuber Viroid Triggers Genome-Wide Changes in Alternative Splicing, Inducible trans-Acting Activity of Phased Secondary Small Interfering RNAs, and Immune Responses. J. Virol., 91, e00247-17.

104. Diener,T.O. (1971) Potato spindle tuber "virus": IV. A replicating, low molecular weight RNA. Virology, 45, 411-428.

105. Itaya,A., Matsuda,Y., Gonzales,R.A., Nelson,R.S. and Ding,B. (2002) Potato spindle tuber viroid Strains of Different Pathogenicity Induces and Suppresses Expression of Common and Unique Genes in Infected Tomato. Mol. Plant-Microbe Interact., 15, 990-999.

106. Shang,X., Cao,Y. and Ma,L. (2017) Alternative Splicing in Plant Genes: A Means of Regulating the Environmental Fitness of Plants. Int. J. Mol. Sci., 18, 432.

107. Jabre,I., Reddy,A.S.N., Kalyna,M., Chaudhary,S., Khokhar,W., Byrne,L.J., Wilson,C.M. and Syed,N.H. (2019) Does co-transcriptional regulation of alternative splicing mediate plant stress responses? Nucleic Acids Res., 47, 2716-2726.

108. Wassenegger,M. and Dalakouras,A. (2021) Viroids as a Tool to Study RNA-Directed DNA Methylation in Plants. Cells, 10, 1187.

109. Wassenegger,M., Heimes,S., Riedel,L. and Sänger,H.L. (1994) RNA-directed de novo methylation of genomic sequences in plants. Cell, 76, 567-76.

110. Castellano,M., Martinez,G., Pallás,V. and Gómez,G. (2015) Alterations in host DNA methylation in response to constitutive expression of Hop stunt viroid RNA in Nicotiana benthamiana plants. Plant Pathol., 64.

111. Cottilli,P., Belda-Palazón,B., Adkar-Purushothama,C.R., Perreault,J.-P., Schleiff,E., Rodrigo,I., Ferrando,A. and Lisón,P. (2019) Citrus exocortis viroid causes ribosomal 
bioRxiv preprint doi: https://doi.org/10.1101/2022.01.06.475203; this version posted January 6,2022 . The copyright holder for this preprint

(which was not certified by peer review) is the author/funder, who has granted bioRxiv a license to display the preprint in perpetuity. It is made available under aCC-BY-ND 4.0 International license.

stress in tomato plants. Nucleic Acids Res., 47, 8649-8661.

112. Bardou,F., Ariel,F., Simpson,C.G., Romero-Barrios,N., Laporte,P., Balzergue,S., Brown,J.W.S. and Crespi,M. (2014) Long Noncoding RNA Modulates Alternative Splicing Regulators in Arabidopsis. Dev. Cell, 30, 166-176. 


\section{FIGURE LEGENDS}

Figure 1: HSVd RNA is detected at $10 \mathrm{dpi}$ in infected plants: A) Representative infected and mock inoculated cucumber plants at the three analyzed time points. Typical plant symptoms characterized by reduction in leaf size and incipient stunting are evident at 24 dpi. Graphic representation of the total transcripts (B) and sRNAs (C) derived from HSVd genome detected in apical leaves at 10, 17 and 24 dpi. D) Scatter plot showing the significant positive correlation (estimated by Pearson correlation coefficient) between the temporal accumulation of vd-transcript and viroid sRNAs (vdsRNAs) in infected cucumber plants.

Figure 2: Analysis of the sRNA population recovered from the analyzed cucumber libraries. A) Principal component analysis based on sRNAs accumulation in biological replicates of HSVd-infected and mock-inoculated plants at the three analyzed time-points. The statistical significance was estimated by Mann-WhitneyWilcoxon test, considering the inter- and intra-group Euclidean distances. B) Diagram showing the means of the temporal relative accumulation and distribution of the total sRNAs reads ranging between 20 and $25 \mathrm{nts}$ (Error bars indicate the SE between biological replicates). The three analized times are represented by colors (green: 10 dpi, orange: $17 \mathrm{dpi}$ and magenta: $24 \mathrm{dpi}$ ). Smooth and striped bars represent data arising from mock-inoculated and HSVd-infected plants. C) Venn diagram representing the number of sRNAs responsive to HSVd infection at 17 and $24 \mathrm{dpi}$. D) Volcano plot representing the endogenous cucumber sRNAs with significant differential expression in infected plants at the three analyzed time points. Blue and red dots indicate up- and down- regulated sRNAs, respectively. The number of differential sRNAs in each time point is detailed. E) Categorization of HSVd-responsive sRNAs. The ball size represents the number of unique reads recovered. The color intensity indicates the sRNA accumulation estimated by the Log2 of the mean of the normalized reads. F) Box-plot analysis showing the general expression value observed for each miRNAfamily member (dots) at 17 and $24 \mathrm{dpi}$. The differential expression of each miRNA family is represented by the median (internal box line) of the LFC values. G) Scatter plot showing the non-significant negative correlation (estimated by Pearson correlation coefficient) between the expression levels of the seven miRNAs responsive to HSVd infection and the accumulation of their targets in infected plants.

Figure 3: Characterization of vd-sRNAs. A) Box plot analysis of the relative accumulation of the total vd-sRNAs (20 to $25 \mathrm{nt}$ ) derived from genomic (plus) and antigenomic (minus) HSVd-RNA strand at the three analyzed time points. B) Distribution of polarity of the total vd-sRNAs discriminated by size. C) Comparative analysis of unique reads of vd-sRNAs. D) Diagram showing the means of the temporal relative accumulation and distribution of the vd-sRNAs reads ranging between 20 and $25 \mathrm{nts}$ (the error bars indicate the SE). The three analized times are represented by colors (green: $10 \mathrm{dpi}$, orange: $17 \mathrm{dpi}$ and magenta: $24 \mathrm{dpi}$ ). E) Graphic representation of the proportion of the 5'- ends in the total recovered vd-sRNAs. The means of the total reads are showed. F) Distribution of the 5'- ends discriminated by size and infection time. G) Genome view of the vd-sRNAs recovered from infected cucumber plants. The vd-sRNAs were plotted according to the position of their 5 '-end onto the HSVd- RNA sequence in either sense (above the $x$-axis) or antisense (below the $x$ axis) configuration. The values on the $y$-axis represent the abundance of total vdsRNAs (sizes indicated by colors) in the three analyzed time point. H) Representation of the temporal accumulation of total vd-sRNAs (means of total vd-sRNAs are represented). Vd-sRNAs recovered in each of the three analized times are represented by colors (green: $10 \mathrm{dpi}$, orange: $17 \mathrm{dpi}$ and magenta: $24 \mathrm{dpi}$ ). I) Representation of the 
vd-sRNA-cleaved transcripts (RTM2 and AQP, upper and lower panel respectively) differentially detected by degradome assay in infected plants. Degradome sequences were plotted onto the predicted targets transcripts. The grey zone indicates the predicted vd-sRNA recognition site (detailed below). The values on the Y-axis represent the relative accumulation of the reads matching onto the $160 \mathrm{nt}$ in length analized region. J) Means of the temporal relative accumulation estimated by sequencing of the target transcripts in infected and control plants (the fine lines indicate the SE). K) Down regulation of the predicted targets mediated by HSVd was validated by transient expression of specific reporters carrying YFP transcriptionally fussed to the RTM2 (upper panel) and AQP (lower panel) target sequences. Reporter expression was determined by fluorescence emission (left) and transcript quantification by RTqPCR (right). Error bars indicate the SE between biological replicates.

Figure 4: Host-transcriptional alterations associated to HSVd-infection. A) Volcano plot representing the differentially expressed transcripts (DET) in infected cucumber plants at 10, 17 and $24 \mathrm{dpi}$. Blue and red dots indicate up- and downregulated transcripts, respectively. DET with LFC $\geq 1$ or $\leq-1$ are represented by bold dots and the number is indicated in brackets. Venn diagram showing all the DET (B) and those with LFC $\geq 1$ or $\leq-1$ (C) identified at 10, 17 and $24 \mathrm{dpi}$ D) Violin plot representing the temporal profile of the transcriptional alteration associated to HSVdinfection. Internal box line indicates the median of the LFC values. E) Gene ontology analysis (plant GOSlim) for DETs identified in HSVd-infected plants at 10, 17 and 24 dpi. Circle size represents level of enrichment and color under- (red) or up-regulated (blue) response. The $-\log (10)$ of the adjusted $P$ values is represented in the $X$-axis. F) Detail of the 21 transcripts differentially expressed at the three analyzed time-points. $G$ ) Clustering analysis of time-course expression profiling of transcripts with differential expression at 10, 17 and 24 dpi. H) Gene ontology analysis (plant GOSlim) for transcripts differentially expressed at both 17 and 24 dpi (LFC $\geq 1$ or $\leq-1)$. I) Venn diagram showing the number of DEU events identified at 10, 17 and $24 \mathrm{dpi}$ and $(\mathrm{J})$ transcripts showing common and specific regulation by differential expression or exon usage. K) Volcano plot representing the events of differential exon usage (DEU) in infected plants at 10, 17 and $24 \mathrm{dpi}$. Blue and red dots indicate up- and downregulated exons, respectively. Events of DEU with LFC $\geq 1$ or $\leq-1$ are represented by bold dots and the number is indicated in brackets.

Figure 5: HSVd induces alterations in the cucumber epigenetic landscape. A) Graphic representation of the total cytosine methylation mean values of HSVd-infected and control plants at 10,17 and 24 dpi (upper panel) and discriminated by sequence context (lower panel). Error bars indicate the standard error between replicates. B) Chromosome view of the temporal evolution of the differential methylation profiles in HSVd-infected plants. Red and blue lines represent hypomethylated and hypermethylated regions, respectively. C) Number of significant hyper- or hypodifferentially methylated regions (DMRs) in the three sequence contexts (CG, CHG and $\mathrm{CHH}$ ) identified in infected plants at 10, 17 and 24 dpi. D) Violin plot representing the temporal profile of the epigenetic alterations (estimated by significant DMRs) associated to HSVd-infection. Internal box line indicates the median of the percentage of differential methylation values. E) Temporal description of the proportion of the cucumber genome regions containing significant DMRs identified in infected plants. F) Global view of the methylation profiles in CG context in ribosomal RNA transcriptional unit at 10, 17 and $24 \mathrm{dpi}$ in HSVd-infected (red) and control (green) plants. In the left part each dot represents the mean methylation values of 50 nucleotides while in the right the total mean methylation values of the whole ribosomal unit are represented. A 
Significantly hypomethylated DMR identified in infected plants at $10 \mathrm{dpi}$ and matching to promoter region is highlighted. G) View of the temporal evolution of the differential methylation in the three sequence contexts ( $\mathrm{CG}, \mathrm{CHG}$ and $\mathrm{CHH}$ ) observed in ribosomal RNA transcriptional unit during infection.

Figure 6: Association between transcriptional alterations and epigenetic changes induced by HSVd-infection. Scatter plots showing the negative correlation (significance estimated by Pearson correlation coefficient) between the expression levels of differential genes containing at least one antagonistic DMR and their global methylation status. A) Considering the three selected gene regions (Promoter/UTR, CDS and intron) and B) considering methylation sequence context. C) Raincloud plots showing the temporal correspondence between the sense and the intensity of the epigenetic changes (upper panels) and the transcriptional activity (lower panels) in response to HSVd-infection. The temporal increase in the intensity of the hypermethylated (upper left) and hypomethylated (upper right) DMRs represented by the mean of the differential methylation (black dots) is associated with an evident decrease (lower right) or increase (lower left), respectively (in intensity) of the transcriptional activity estimated by the LFC mean values (black dots).

Figure 7: Graphic representation showing the temporal evolution of the global host-regulatory response to HSVd-infection in cucumber plants. The predominant regulatory layers affected in each analyzed infection time are hierarchical represented by the density (box length) estimated by the number of alterations and intensity (box color strength) estimated by the absolute differential value of the response. To represent the density of the alterations in methylation we considered the number of genes with significant DMRs in CG context. In the sRNA group are included all identified classes except rib-sRNAs and miRNAs. The global position in each analyzed time-point indicates the predominance of the regulatory layer in the global response to infection. Below is also represented the relative accumulation (estimated by transcript recovering) of HSVd in infected plants.

\section{SUPPLEMENTAL FIGURES}

Supplemental Figure S1: Graphic representation of the experimental approach used in this study to identify HSVd-induced alterations in the cucumber transcriptome, sRNAnome and methylome.

Supplemental Figure S2: Viroid-derived sRNAs arise from both HSVd-RNA strands.

Supplemental Figure S3: The accumulation of 21 and 24 nt vd-sRNAs evolves differentially during the infection.

Supplemental Figure S4: Genome view of the vd-sRNAs recovered from infected cucumber plants.

Supplemental Figure S5: Two HSVd regions show extremely low accumulation of vdsRNAs.

Supplemental Figure S6: Details of the pipeline used for the degradome analysis to identify sequences indicative of vd-sRNA-mediated cleavage of cucumber transcripts.

Supplemental Figure S7: Representation of the cellulose synthase transcript differentially detected to be cleaved by a vd-sRNA by degradome assay in infected plants. 
bioRxiv preprint doi: https://doi.org/10.1101/2022.01.06.475203; this version posted January 6,2022 . The copyright holder for this preprint

(which was not certified by peer review) is the author/funder, who has granted bioRxiv a license to display the preprint in perpetuity. It is made available under aCC-BY-ND 4.0 International license.

Supplemental Figure S8: Details of the pipeline used to identify non-annotated differentially expressed cucumber transcripts.

Supplemental Figure S9: Gene ontology analysis (plant GOSlim) for transcripts with differential exon usage identified in HSVd-infected plants at 10, 17 and $24 \mathrm{dpi}$.

Supplemental Figure S10: Global view of the temporal evolution of the differential methylation in ribosomal genes in $\mathrm{CHG}$ and $\mathrm{CHH}$ contexts.

Supplemental Figure S11: Association between HSVd accumulation and rDNA methylation.

Supplemental Figure S12: Association between transcriptional alterations and epigenetic changes induced by HSVd-infection.

Supplemental Figure S13: HSVd-infection is associated to significant transcriptional alterations of rRNA. 